\title{
Nonlinear Schrödinger equations and their spectral semi-discretizations over long times
}

\author{
Ludwig Gauckler ${ }^{1}$ and Christian Lubich ${ }^{1}$
}

July 7, 2008

${ }^{1}$ Mathematisches Institut, Universität Tübingen, Auf der Morgenstelle 10, D-72076 Tübingen, Germany.
email: \{gauckler,lubich\}@na.uni-tuebingen.de

\begin{abstract}
Cubic Schrödinger equations with small initial data (or small nonlinearity) and their spectral semi-discretizations in space are analyzed. It is shown that along both, the solution of the nonlinear Schrödinger equation as well as the solution of the semi-discretized equation, the actions of the linear Schrödinger equation are approximately conserved over long times. This also allows to show approximate conservation of energy and momentum along the solution of the semi-discretized equation over long times. These results are obtained by analyzing a modulated Fourier expansion in time.

They are valid in arbitrary spatial dimension.
\end{abstract}

Keywords: Nonlinear Schrödinger equation - Spectral semi-discretization - Long-time behavior - Near-conservation of actions, energy, and momentum - Modulated Fourier expansion

MSC (2000): 37K55, 65M70

\section{Introduction}

We consider the nonlinear Schrödinger equation

$$
i u_{t}=-\Delta u+V * u+|u|^{2} u
$$

with periodic boundary conditions in dimension $d \geq 1$, where $u=u(x, t), x \in \mathbb{T}^{d}=\mathbb{R}^{d} / 2 \pi \mathbb{Z}^{d}$, $t \geq 0$. The linear potential $V=V(x) \in L^{2}\left(\mathbb{T}^{d}\right)$ acts by convolution on $u$. It is assumed to be periodic with real Fourier coefficients.

Such equations have been studied by Bambusi and Grébert [3], Bourgain [4], and Eliasson and Kuksin [7]. Bambusi and Grébert transformed them to a normal form, which allows to show approximate conservation of the actions of the linear Schrödinger equation $i u_{t}=-\Delta u+V * u$ along solutions of (1) over long times in case of small initial data [3, Theorem 3.25]. Here instead, we prove such a result using the alternative technique of modulated Fourier expansions. This technique of proof has the advantage of being transferable to discretizations of the equation. Here we show that the approximate conservation of actions remains true after a spectral semi-discretization in space. Moreover, we show that the energy and the momentum of (1) are approximately conserved along solutions of the semi-discretized equation. Similar results for a full discretization of (1) using in addition a Lie-Trotter splitting in time are shown in a separate paper [8].

The technique of modulated Fourier expansions has been used by Hairer and Lubich [9] to study conservation properties of numerical methods for highly oscillatory ordinary differential equations, see also [11, Chapter XIII]. Recently, together with Cohen, they extended this technique to semilinear wave equations [6], their spectral semi-discretizations in space [10], and full discretizations with trigonometric integrators and the Störmer-Verlet method [5].

In Section 2 we state the result of approximate conservation of actions for the solution of (1). For the proof of this result we study a modulated Fourier expansion in Section 3 and conservation 
properties of this expansion in Section 4. In Sections 5 and 6 we extend the results to the standard spectral discretization in space of (1) and study the long-time near-conservation of energy and momentum.

\section{Near-conservation of actions for the nonlinear Schröding- er equation}

\section{$2.1 \quad$ Statement of the result}

In this section we formulate our main result for solutions of the nonlinear Schrödinger equation (1). To motivate this result we first consider the linear Schrödinger equation $i u_{t}=-\Delta u+V * u$ and note that the actions

$$
I_{j}(u, \bar{u})=\frac{1}{2}\left|u_{j}\right|^{2} \quad\left(j \in \mathbb{Z}^{d}\right)
$$

are exactly conserved along any solution of this equation. Here $u_{j}=\mathcal{F}_{j}(u)$ denotes the $j$-th Fourier coefficient of a periodic function $u=\sum_{j \in \mathbb{Z}^{d}} u_{j} e^{i(j \cdot x)}$ where $j \cdot x=j_{1} x_{1}+\cdots+j_{d} x_{d}$. In fact the linear Schrödinger equation as an equation for the Fourier coefficients reads $i\left(u_{j}\right)_{t}=\omega_{j} u_{j}$, where

$$
\omega_{j}=|j|^{2}+\mathcal{F}_{j}(V) \quad\left(j \in \mathbb{Z}^{d}\right)
$$

are the frequencies with $|j|^{2}=j_{1}^{2}+\cdots+j_{d}^{2}$. The frequencies behave asymptotically like $|j|^{2}$.

Our main result states that along solutions of the nonlinear equation (1) the actions (2) are approximately conserved over long times provided that the frequencies satisfy a non-resonance condition and that the initial data is small. The smallness of the initial data is measured in the Sobolev norm

$$
\|u\|_{s}=\left(\sum_{j \in \mathbb{Z}^{d}}\left|\omega_{j}\right|^{s}\left|u_{j}\right|^{2}\right)^{\frac{1}{2}}
$$

for $s \geq 0$. In this definition $\omega_{j}$ is replaced by 1 in case of $\omega_{j}=0$. Because of the asymptotics of the frequencies the norm $\|\cdot\|_{s}$ is equivalent to the Sobolev norm of $H^{s}$. The $s$-norm of the initial data is assumed to be of size $\varepsilon \ll 1$. Equivalent to the condition of small initial data we could require a small nonlinearity and initial data of size 1 in the norm $\|\cdot\|_{s}$ by replacing (1) by $i u_{t}=-\Delta u+V * u+\varepsilon^{2}|u|^{2} u$. We consider the almost-conservation of actions on time intervals of length $\varepsilon^{-N}$ for natural numbers $N$.

For the precise statement of the non-resonance condition on the frequencies (3) of the linear part of the equation we introduce the following notations similar to [6]. For a sequence $\mathbf{k}=\left(k_{l}\right)_{l \in \mathbb{Z}^{d}}$ of integers $k_{l}$ and the sequence $\boldsymbol{\omega}=\left(\omega_{l}\right)_{l \in \mathbb{Z}^{d}}$ of frequencies $(3)$ we write

$$
j(\mathbf{k})=\sum_{l \in \mathbb{Z}^{d}} k_{l} l, \quad\|\mathbf{k}\|=\sum_{l \in \mathbb{Z}^{d}}\left|k_{l}\right|, \quad \mathbf{k} \cdot \boldsymbol{\omega}=\sum_{l \in \mathbb{Z}^{d}} k_{l} \omega_{l}, \quad \boldsymbol{\omega}^{\sigma|\mathbf{k}|}=\prod_{l \in \mathbb{Z}^{d}} \omega_{l}^{\sigma\left|k_{l}\right|}
$$

for $\sigma \in \mathbb{R}$. In our analysis we have to divide by $\mathbf{k} \cdot \boldsymbol{\omega}-\omega_{j(\mathbf{k})}$. We collect pairs $(j(\mathbf{k}), \mathbf{k})$ with small denominator in the set of near-resonant indices

$$
\mathcal{R}_{\varepsilon}=\left\{(j, \mathbf{k}): j=j(\mathbf{k}), \mathbf{k} \neq\langle j\rangle,\left|\mathbf{k} \cdot \boldsymbol{\omega}-\omega_{j}\right|<\varepsilon^{\frac{1}{2}},\|\mathbf{k}\| \leq 2 N+2\right\},
$$

where $\langle j\rangle=\left(\delta_{j l}\right)_{l \in \mathbb{Z}^{d}}$ with Kronecker's delta, and impose on this set the non-resonance condition

$$
\sup _{(j, \mathbf{k}) \in \mathcal{R}_{\varepsilon}} \frac{\left|\omega_{j}\right|^{s-\frac{d+1}{2}}}{\left|\boldsymbol{\omega}^{\left(s-\frac{d+1}{2}\right)|\mathbf{k}|}\right|} \varepsilon^{\|\mathbf{k}\|+1} \leq C_{0} \varepsilon^{2 N+4}
$$

for a constant $C_{0}$ (independent of $\varepsilon$ ) and a given natural number $N$. Here again and in the following whenever the absolute value of the frequencies appears, zero frequencies are replaced by 1 . The proof of the following theorem will be the subject of Sections 3 and 4 . 
Theorem 1. For given $N$ and $s \geq d+1$ there exists $\varepsilon_{0}>0$ such that the following holds: Under the conditions of small initial data $\|u(\cdot, 0)\|_{s} \leq \varepsilon \leq \varepsilon_{0}$ and of non-resonance (5), the estimate

$$
\sum_{l \in \mathbb{Z}^{d}}\left|\omega_{l}\right|^{s} \frac{\left|I_{l}(u(\cdot, t), \bar{u}(\cdot, t))-I_{l}(u(\cdot, 0), \bar{u}(\cdot, 0))\right|}{\varepsilon^{2}} \leq C \varepsilon^{\frac{3}{2}} \quad \text { for } 0 \leq t \leq \varepsilon^{-N}
$$

holds for solutions $u(x, t)$ of (1) with a constant $C$ which depends on $C_{0}, d, N, s$, and $V$ but is independent of $\varepsilon$ and $t$.

This theorem slightly refines [3, Theorem 3.26]. We mention that Theorem 1 (and also Theorem 2 below) can be extended easily to nonlinear Schrödinger equations with nonlinearities of the form $g\left(|u|^{2}\right)|u|^{2} u$ where $g$ is a polynomial (the constants will then depend also on $g$ ).

\subsection{On the non-resonance condition}

We now show that the non-resonance condition (5) is realistic in the sense that it is fulfilled for a large set of potentials $V$. We do this by proving that our non-resonance condition is implied by the one used by Bambusi and Grébert [3]. They show the following proposition.

Proposition 1 (Bambusi and Grébert [3, Theorem 3.22]). Fix $m>\frac{d}{2}$ and $R>0$. The space

$$
\left\{V(x)=\sum_{j \in \mathbb{Z}^{d}} v_{j} e^{i(j \cdot x)}:\left|v_{j}(1+|j|)^{m} / R\right| \leq \frac{1}{2} \text { for all } j\right\}
$$

endowed with the product probability measure has a subset $\mathcal{S}$ of measure 1 such that for any $V \in \mathcal{S}$ the following property holds. For any $r>0$ there exist $\gamma>0$ and $\alpha>0$ such that for any $L \geq 1$,

$$
\left|\sum_{j \in \mathbb{Z}^{d}} \omega_{j} k_{j}\right| \geq \frac{\gamma}{L^{\alpha}}
$$

for any sequence $\mathbf{k}=\left(k_{l}\right)_{l \in \mathbb{Z}}$ of integers $k_{l}$ fulfilling $0 \neq\|\mathbf{k}\| \leq r+2$ and $\sum_{|j|>L}\left|k_{j}\right| \leq 2$ except if $\mathbf{k}=\langle j\rangle-\langle l\rangle$ with $|j|=|l|$.

We remark that the exception in Bambusi's and Grébert's original version of Proposition 1 covers those $\mathbf{k}$ with $k_{j}=0$ for $|j| \leq L^{\sqrt{\alpha / m}}$ and $\sum_{|j|=n} k_{j}=0$ for all $n>L \sqrt{\alpha / m}$. Note however that we can assume $\alpha \geq m$ as becomes clear from the proof presented in [3] and is also used there, and hence Proposition 1 as stated above is indeed implied by [3, Theorem 3.22].

Proposition 2. Fix N. For sufficiently large s, the non-resonance condition (5) holds for all $V \in \mathcal{S}$, where $\mathcal{S}$ is the set of Proposition 1, with a constant $C_{0}$ which depends only on $N$, s, and $V$.

Proof. We use the notations of Proposition 1. Let $V \in \mathcal{S},(j, \mathbf{k}) \in \mathcal{R}_{\varepsilon}$, and $\|\mathbf{k}\| \leq r+1$. We write $\mathbf{k} \cdot \boldsymbol{\omega}=k_{l} \omega_{l}+\sum_{|j| \leq L, j \neq l} k_{j} \omega_{j}$ with $|l| \geq L$ and $L \geq 1$ minimal. We have $\mathbf{k} \neq\langle l\rangle$ since otherwise $l=j(\mathbf{k})=j$ and $\mathbf{k}=\langle j\rangle$ contradictory to $(j, \mathbf{k}) \in \mathcal{R}_{\varepsilon}$. We also have $\mathbf{k} \neq\langle j\rangle+(\langle l\rangle-\langle m\rangle)$ since otherwise $j+(l-m)=j(\mathbf{k})=j$ and hence again $\mathbf{k}=\langle j\rangle$. Hence, $\mathbf{k}-\langle j\rangle$ is not an exception in Proposition 1. This proposition then yields $\frac{\gamma}{L^{\alpha}} \leq\left|\mathbf{k} \cdot \boldsymbol{\omega}-\omega_{j}\right|<\varepsilon^{\frac{1}{2}}$. The statement now follows as in the proof of [6, Lemma 1].

Note that the non-resonance condition of Bambusi and Grébert in Proposition 1 requires that all frequencies are nonzero. Our non-resonance condition (5) does not impose this restriction.

\section{Modulated Fourier expansions}

The analysis of the solution of (1) is done by the method of modulated Fourier expansions. We follow the lines of $[6]$.

Throughout this section we work under the assumptions of Theorem 1. All appearing constants will be denoted by $C$. The main point is that all these constants do not depend on $\varepsilon$ and the time $0 \leq t \leq \varepsilon^{-1}$; however, they may depend on $C_{0}$ and $N$ from the non-resonance condition (5), the dimension $d$, the regularity parameter $s$, and the potential $V$. 


\subsection{The modulation system}

We are looking for a function $\tilde{u}(x, t)$ which approximates the solution $u(x, t)$ of $(1)$ by a modulated Fourier expansion

$$
\tilde{u}(x, t)=\sum_{\|\mathbf{k}\| \leq K} z_{j(\mathbf{k})}^{\mathbf{k}}(\varepsilon t) e^{i(j(\mathbf{k}) \cdot x)} e^{-i(\mathbf{k} \cdot \boldsymbol{\omega}) t}=\sum_{\|\mathbf{k}\| \leq K} z^{\mathbf{k}}(x, \varepsilon t) e^{-i(\mathbf{k} \cdot \boldsymbol{\omega}) t}
$$

with $z^{\mathbf{k}}(x, \varepsilon t)=z_{j(\mathbf{k})}^{\mathbf{k}}(\varepsilon t) e^{i(j(\mathbf{k}) \cdot x)}$. We set $z_{l}^{\mathbf{k}}(\varepsilon t)=0$ for $l \neq j(\mathbf{k})$. In contrast to [6] the functions $z^{\mathbf{k}}$ consist of a single wave. This is crucial for the validity of Theorem 1 in arbitrary dimension as is seen in the proof of Proposition 2. In the following we choose $K=2 N+2$ and tacitly assume $\|\mathbf{k}\| \leq K$ unless stated otherwise. We insert (6) in (1) and note that for $\mathbf{k}=\mathbf{k}^{1}+\mathbf{k}^{2}-\mathbf{k}^{3}$ we have

$$
\mathcal{F}_{j}\left(z^{\mathbf{k}^{1}} z^{\mathbf{k}^{2}} \overline{z^{\mathbf{k}^{3}}}\right)= \begin{cases}z_{j\left(\mathbf{k}^{1}\right)}^{\mathbf{k}^{1}} z_{j\left(\mathbf{k}^{2}\right)}^{\mathbf{k}^{2}} \overline{z_{j\left(\mathbf{k}^{3}\right)}^{\mathbf{k}^{3}}}, & j=j(\mathbf{k}), \\ 0, & \text { else }\end{cases}
$$

since $j(\mathbf{k})=j\left(\mathbf{k}^{1}\right)+j\left(\mathbf{k}^{2}\right)-j\left(\mathbf{k}^{3}\right)$. If we compare the coefficients of $e^{i(j(\mathbf{k}) \cdot x)} e^{-i(\mathbf{k} \cdot \boldsymbol{\omega}) t}$ we thus arrive at the modulation system

$$
i \varepsilon \dot{z}_{j(\mathbf{k})}^{\mathbf{k}}+(\mathbf{k} \cdot \boldsymbol{\omega}) z_{j(\mathbf{k})}^{\mathbf{k}}=\omega_{j(\mathbf{k})} z_{j(\mathbf{k})}^{\mathbf{k}}+\sum_{\mathbf{k}^{1}+\mathbf{k}^{2}-\mathbf{k}^{3}=\mathbf{k}} \mathcal{F}_{j(\mathbf{k})}\left(z^{\mathbf{k}^{1}} z^{\mathbf{k}^{2} \overline{z^{\mathbf{k}^{3}}}}\right) .
$$

Here, we denote by $\dot{z}_{j(\mathbf{k})}^{\mathbf{k}}$ the derivative with respect to $\tau=\varepsilon t$. Requiring $\tilde{u}(\cdot, 0)=u(\cdot, 0)$ further yields

$$
\sum_{\mathbf{k}} z_{j}^{\mathbf{k}}(0)=u_{j}(0)
$$

\subsection{Results on the modulation functions}

We will construct an approximate solution of the modulation system (7) for $0 \leq \varepsilon t=\tau \leq 1$. For measuring the size of functions $\mathbf{z}=\left(z^{\mathbf{k}}\right)_{\mathbf{k}}=\left(z_{j(\mathbf{k})}^{\mathbf{k}} e^{i(j(\mathbf{k}) \cdot x)}\right)_{\mathbf{k}}$ we use the norm

$$
\|\mathbf{z}\|_{s}=\left\|\sum_{\mathbf{k}}\left\{z^{\mathbf{k}}\right\}\right\|_{s}=\left(\sum_{j \in \mathbb{Z}^{d}}\left|\omega_{j}\right|^{s}\left(\sum_{\mathbf{k}}\left|z_{j}^{\mathbf{k}}\right|\right)^{2}\right)^{\frac{1}{2}}
$$

where

$$
\{v\}(x)=\sum_{j \in \mathbb{Z}^{d}}\left|v_{j}\right| e^{i(j \cdot x)}
$$

for a periodic function $v(x)=\sum_{j \in \mathbb{Z}^{d}} v_{j} e^{i(j \cdot x)}$. This norm yields a mixture between the $l^{2}$-based framework of [6] and a more handy $l^{1}$-based framework. We use the notation $\hat{\imath}$ for the scaling

$$
\hat{\mathbf{z}}=\left(\hat{z}^{\mathbf{k}}\right)_{\mathbf{k}}=\left(\left|\boldsymbol{\omega}^{\frac{2 s-d-1}{4}|\mathbf{k}|}\right| z^{\mathbf{k}}\right)_{\mathbf{k}}
$$

and prove the following proposition for the approximate solution.

Proposition 3. There exists a function $\tilde{u}(x, t)=\sum_{\|\mathbf{k}\| \leq 2 N+2} z_{j(\mathbf{k})}^{\mathbf{k}}(\varepsilon t) e^{i(j(\mathbf{k}) \cdot x)} e^{-i(\mathbf{k} \cdot \boldsymbol{\omega}) t}$ for $x \in \mathbb{T}^{d}$ and $0 \leq \varepsilon t \leq 1$ satisfying

$$
\|u(\cdot, t)-\tilde{u}(\cdot, t)\|_{s} \leq C \varepsilon^{N+2} .
$$

Moreover, the following estimates hold:

- $\tilde{u}$ is small,

$$
\|\tilde{u}(\cdot, t)\|_{s} \leq C \varepsilon
$$

- $\mathbf{z}$ is small,

$$
\begin{gathered}
z_{j(\mathbf{k})}^{\mathbf{k}}=0 \text { for }(j(\mathbf{k}), \mathbf{k}) \in \mathcal{R}_{\varepsilon}, \quad\|\hat{\mathbf{z}}\| \|_{\frac{d+1}{2}} \leq C \varepsilon, \quad \sum_{j \in \mathbb{Z}^{d}}\left|\omega_{j}\right|^{s}\left|z_{j}^{\langle j\rangle}\right|^{2} \leq C \varepsilon^{2}, \\
\sum_{j \in \mathbb{Z}^{d}}\left|\omega_{j}\right|^{s}\left(\sum_{\mathbf{k} \neq\langle j\rangle}\left|z_{j}^{\mathbf{k}}\right|\right)^{2} \leq C \varepsilon^{5}, \quad \sum_{j \in \mathbb{Z}^{d}}\left|\omega_{j}\right|^{\frac{d+1}{2}}\left(\sum_{\mathbf{k} \neq\langle j\rangle}\left|\hat{z}_{j}^{\mathbf{k}}\right|\right)^{2} \leq C \varepsilon^{5} .
\end{gathered}
$$


- $\mathbf{z}$ is a solution of (7a) up to a small defect $\mathbf{d}=\mathbf{e}+\mathbf{f}$ with $e_{j}^{\mathbf{k}}=0$ for $j \neq j(\mathbf{k})$ or $(j, \mathbf{k}) \in \mathcal{R}_{\varepsilon}$ and $f_{j}^{\mathbf{k}}=0$ for $(j, \mathbf{k}) \notin \mathcal{R}_{\varepsilon}$,

$$
\|\mathbf{e}\|_{s} \leq C \varepsilon^{N+3}, \quad\|\hat{\mathbf{e}}\|_{\frac{d+1}{2}} \leq C \varepsilon^{N+3} .
$$

All constants are independent of $\varepsilon$ and $0 \leq t \leq \varepsilon^{-1}$ but may depend on $C_{0}, d, N, s$, and $V$.

The proof of this proposition will cover the remaining part of this section except Subsection 3.9. At a first reading it might be useful to skip this highly technical part.

\subsection{Iterative solution of the modulation system}

The pairs $(j,\langle j\rangle)$ play a special role since $\mathbf{k} \cdot \boldsymbol{\omega}-\omega_{j}=0$ for $\mathbf{k}=\langle j\rangle$. We therefore collect those pairs $(j(\mathbf{k}), \mathbf{k})$ which are not of this form and are not near-resonant in the set

$$
\mathcal{S}_{\varepsilon}=\left\{(j, \mathbf{k}): j=j(\mathbf{k}), \mathbf{k} \neq\langle j\rangle,(j, \mathbf{k}) \notin \mathcal{R}_{\varepsilon},\|\mathbf{k}\| \leq K\right\} .
$$

The solution of the modulation system (7) is determined up to a small defect by an iterative procedure as in [6]. We start by setting

$$
\left[z_{j}^{\langle j\rangle}\right]^{0}=u_{j}(0) \text { and }\left[z_{j(\mathbf{k})}^{\mathbf{k}}\right]^{0}=0 \text { for } \mathbf{k} \neq\langle j(\mathbf{k})\rangle
$$

for $0 \leq \varepsilon t=\tau \leq 1$. For $n \geq 0$ and $0 \leq \varepsilon t=\tau \leq 1$ we set, motivated by isolating the dominant terms in (7a),

$$
\begin{aligned}
& {\left[z_{j}^{\mathbf{k}}\right]^{n+1}=\frac{1}{\mathbf{k} \cdot \boldsymbol{\omega}-\omega_{j}}\left[-i \varepsilon \dot{z}_{j}^{\mathbf{k}}+\sum_{\mathbf{k}^{1}+\mathbf{k}^{2}-\mathbf{k}^{3}=\mathbf{k}} \mathcal{F}_{j}\left(z^{\mathbf{k}^{1}} z^{\mathbf{k}^{2}} \overline{z^{\mathbf{k}^{3}}}\right)\right]^{n} \quad \text { for }(j, \mathbf{k}) \in \mathcal{S}_{\varepsilon},} \\
& {\left[\dot{z}_{j}^{\langle j\rangle}\right]^{n+1}=-i \varepsilon^{-1}\left[\sum_{\mathbf{k}^{1}+\mathbf{k}^{2}-\mathbf{k}^{3}=\langle j\rangle} \mathcal{F}_{j}\left(z^{\mathbf{k}^{1}} z^{\mathbf{k}^{2}} \overline{z^{\mathbf{k}^{3}}}\right)\right]^{n}, \quad\left[z_{j}^{\langle j\rangle}(0)\right]^{n+1}=u_{j}(0)-\left[\sum_{\mathbf{k} \neq\langle j\rangle} z_{j}^{\mathbf{k}}(0)\right]^{n},} \\
& {\left[z_{j}^{\mathbf{k}}\right]^{n+1}=0 \text { for }(j, \mathbf{k}) \in \mathcal{R}_{\varepsilon} .}
\end{aligned}
$$

The notation $[\cdot]^{n}$ means that the $n$-th iterates of the variables within the brackets are taken. In each iteration step we have initial value problems for $z_{j}^{\langle j\rangle}$ and algebraic equations for the other $z_{j}^{\mathbf{k}}$. We stop the iteration after $L=2 N+2$ steps. The functions of Proposition 3 will be $\mathbf{z}=[\mathbf{z}]^{L}$.

\subsection{Abstract formulation of the iteration}

We set

$$
[[\mathbf{k}]]= \begin{cases}\max \left(\frac{1}{2}(\|\mathbf{k}\|+1), 2\right), & \mathbf{k} \neq\langle j\rangle, \\ \frac{1}{2}(\|\mathbf{k}\|+1)=1, & \mathbf{k}=\langle j\rangle .\end{cases}
$$

We split and scale the variables as follows:

$$
a_{j}^{\mathbf{k}}=\left\{\begin{array}{ll}
\varepsilon^{-[[\mathbf{k}]]} z_{j}^{\mathbf{k}}, & \mathbf{k}=\langle j\rangle, \\
0, & \mathbf{k} \neq\langle j\rangle
\end{array} \quad \text { and } \quad b_{j}^{\mathbf{k}}= \begin{cases}0, & \mathbf{k}=\langle j\rangle, \\
\varepsilon^{-[[\mathbf{k}]]} z_{j}^{\mathbf{k}}, & \mathbf{k} \neq\langle j\rangle,\end{cases}\right.
$$

and write $\mathbf{a}=\left(a^{\mathbf{k}}\right)_{\mathbf{k}}=\left(a_{j(\mathbf{k})}^{\mathbf{k}} e^{i(j(\mathbf{k}) \cdot x)}\right)_{\mathbf{k}}, \mathbf{b}=\left(b^{\mathbf{k}}\right)_{\mathbf{k}}=\left(b_{j(\mathbf{k})}^{\mathbf{k}} e^{i(j(\mathbf{k}) \cdot x)}\right)_{\mathbf{k}}$, and $\mathbf{c}=\mathbf{a}+\mathbf{b}$. We further define

$$
(\boldsymbol{\Omega c})_{j}^{\mathbf{k}}= \begin{cases}\left(\mathbf{k} \cdot \boldsymbol{\omega}-\omega_{j}\right) c_{j}^{\mathbf{k}}, & (j, \mathbf{k}) \in \mathcal{S}_{\varepsilon}, \\ \varepsilon^{\frac{1}{2}} c_{j}^{\mathbf{k}}, & \text { else }\end{cases}
$$

and

$$
\mathbf{F}(\mathbf{c})_{j}^{\mathbf{k}}=\varepsilon^{-\max ([[\mathbf{k}]], 2)} \sum_{\mathbf{k}^{1}+\mathbf{k}^{2}-\mathbf{k}^{3}=\mathbf{k}} \mathcal{F}_{j}\left(\varepsilon^{\left[\left[\mathbf{k}^{1}\right]\right]+\left[\left[\mathbf{k}^{2}\right]\right]+\left[\left[\mathbf{k}^{3}\right]\right]} c^{\mathbf{k}^{2}} c^{\mathbf{k}^{2}} c^{\mathbf{k}^{3}}\right) .
$$


The iteration in the rescaled variables becomes

$$
\begin{aligned}
& {\left[b_{j}^{\mathbf{k}}\right]^{n+1}=\left[-i \varepsilon\left(\boldsymbol{\Omega}^{-1} \dot{\mathbf{b}}\right)_{j}^{\mathbf{k}}\right]^{n}+\left[\left(\boldsymbol{\Omega}^{-1} \mathbf{F}(\mathbf{c})\right)_{j}^{\mathbf{k}}\right]^{n} \text { for }(j, \mathbf{k}) \in \mathcal{S}_{\varepsilon},} \\
& {\left[\dot{a}_{j}^{\langle j\rangle}\right]^{n+1}=-i\left[\mathbf{F}(\mathbf{c})_{j}^{\langle j\rangle}\right]^{n}, \quad\left[a_{j}^{\langle j\rangle}(0)\right]^{n+1}=\varepsilon^{-1} u_{j}(0)-\left[\sum_{\mathbf{k} \neq\langle j\rangle} \varepsilon^{[[\mathbf{k}]]-1} b_{j}^{\mathbf{k}}(0)\right]^{n} .}
\end{aligned}
$$

We also use a second rescaling of the variables, $\hat{a}_{j}^{\mathbf{k}}=\left|\omega^{\frac{2 s-d-1}{4}}\right| \mathbf{k}|| a_{j}^{\mathbf{k}}, \hat{b}_{j}^{\mathbf{k}}=\left|\boldsymbol{\omega}^{\frac{2 s-d-1}{4}|\mathbf{k}|}\right| b_{j}^{\mathbf{k}}$, and $\hat{\mathbf{c}}=\hat{\mathbf{a}}+\hat{\mathbf{b}}$. With

$$
\begin{aligned}
\hat{\mathbf{F}}(\hat{\mathbf{c}})_{j}^{\mathbf{k}}= & \varepsilon^{-\max ([[\mathbf{k}], 2)}\left|\boldsymbol{\omega}^{\frac{2 s-d-1}{4}|\mathbf{k}|}\right| \\
& \sum_{\mathbf{k}^{1}+\mathbf{k}^{2}-\mathbf{k}^{3}=\mathbf{k}} \mathcal{F}_{j}\left(\varepsilon^{\left[\left[\mathbf{k}^{1}\right]\right]+\left[\left[\mathbf{k}^{2}\right]\right]+\left[\left[\mathbf{k}^{3}\right]\right]}\left|\boldsymbol{\omega}^{-\frac{2 s-d-1}{4}\left(\left|\mathbf{k}^{1}\right|+\left|\mathbf{k}^{2}\right|+\left|\mathbf{k}^{3}\right|\right)}\right| \hat{\mathbf{k}^{1}}{ }^{1} \mathbf{k}^{2} \overline{\hat{c}^{3}}\right)
\end{aligned}
$$

the iteration for $\hat{\mathbf{b}}$ becomes

$$
\left[\hat{b}_{j}^{\mathbf{k}}\right]^{n+1}=\left[-i \varepsilon\left(\boldsymbol{\Omega}^{-1} \dot{\hat{\mathbf{b}}}\right)_{j}^{\mathbf{k}}\right]^{n}+\left[\left(\boldsymbol{\Omega}^{-1} \hat{\mathbf{F}}(\hat{\mathbf{c}})\right)_{j}^{\mathbf{k}}\right]^{n} \quad \text { for }(j, \mathbf{k}) \in \mathcal{S}_{\varepsilon} .
$$

\subsection{Estimating the nonlinearity}

The following lemma is crucial for estimations of the nonlinearity. It reflects the fact that for $s>\frac{d}{2}$ the Sobolev space $H^{s}$ is an algebra.

Lemma 1. We have for $s>\frac{d}{2}$

$$
\|\bar{v}\|_{s} \leq C\|v\|_{s}, \quad\|v w\|_{s} \leq C\|v\|_{s}\|w\|_{s}, \quad \text { and } \quad\left\|\sum_{\mathbf{k}, \mathbf{l}}\left\{c^{\mathbf{k}} d^{\mathbf{l}}\right\}\right\|_{s} \leq C\left\|\sum_{\mathbf{k}}\left\{c^{\mathbf{k}}\right\}\right\|_{s}\left\|\sum_{\mathbf{l}}\left\{d^{\mathbf{l}}\right\}\right\|_{s}
$$

with a constant $C$ which depends on $d, s$, and $V$ but is independent of $\varepsilon$.

Proof. The first inequality is clear from the asymptotics of the frequencies. Using the CauchySchwarz inequality we get

$$
\|v w\|_{s}^{2} \leq \sum_{j \in \mathbb{Z}^{d}}\left|\omega_{j}\right|^{s}\left(\sum_{k+l=j}\left|v_{k}\right|\left|w_{l}\right|\right)^{2} \leq \sum_{j \in \mathbb{Z}^{d}}\left(\sum_{k+l=j}\left|\omega_{k}\right|^{s}\left|v_{k}\right|^{2}\left|\omega_{l}\right|^{s}\left|w_{l}\right|^{2}\right)\left(\sum_{k+l=j} \frac{\left|\omega_{j}\right|^{s}}{\left|\omega_{k} \omega_{l}\right|^{s}}\right) .
$$

The term $\sum_{k+l=j} \frac{\left|\omega_{j}\right|^{s}}{\left|\omega_{k} \omega_{l}\right|^{s}}$ can be bounded independently of $j$ by $C \sum_{0 \neq k \in \mathbb{Z}^{d}} \frac{1}{|k|^{2 s}}$ where $C$ depends on $V$. We replace the Euclidean norm $|\cdot|$ in the latter series by the equivalent 1-norm $|k|_{1}=$ $\left|k_{1}\right|+\cdots+\left|k_{d}\right|$ (the constant then will also depend on $d$ ). By counting the vectors $k \in \mathbb{Z}^{d}$ with 1-norm equal to a given number $n$ we see that this series converges for $2 s>d$, cf. [2, Proof of Theorem 4 in $\S 24]$. This gives the second estimate of (11). For the third estimate we just notice

$$
\begin{aligned}
\left\|\sum_{\mathbf{k}, \mathbf{l}}\left\{c^{\mathbf{k}} d^{\mathbf{l}}\right\}\right\|_{s}^{2} & =\sum_{j \in \mathbb{Z}^{d}}\left|\omega_{j}\right|^{s}\left(\sum_{\mathbf{k}, \mathbf{l}}\left|\mathcal{F}_{j}\left(c^{\mathbf{k}} d^{\mathbf{l}}\right)\right|\right)^{2} \\
& \leq \sum_{j \in \mathbb{Z}^{d}}\left|\omega_{j}\right|^{s}\left(\sum_{k+l=j} \sum_{\mathbf{k}}\left|c_{k}^{\mathbf{k}}\right| \sum_{\mathbf{l}}\left|d_{l}^{\mathbf{l}}\right|\right)^{2}=\left\|\sum_{\mathbf{k}, \mathbf{l}}\left\{c^{\mathbf{k}}\right\}\left\{d^{\mathbf{l}}\right\}\right\|_{s}^{2} .
\end{aligned}
$$

The second estimate of Lemma 1 is well known, see for example [1, Theorem 5.23]. The proof presented here does not make use of the Sobolev embedding theorem as in [1]. However, the proof presented here is in essence well known.

Now, we can study the nonlinearity.

Lemma 2. We have

$$
\begin{aligned}
\left\|\boldsymbol{\Omega}^{-1} \mathbf{c}\right\|_{s} & \leq \varepsilon^{-\frac{1}{2}}\|\mathbf{c}\|_{s} \\
\|\mathbf{F}(\mathbf{c})\|_{s} & \leq C \varepsilon\|\mathbf{c}\|_{s}^{3} \\
\|\mathbf{F}(\mathbf{c})-\mathbf{F}(\tilde{\mathbf{c}})\|_{s} & \leq C \varepsilon\|\mathbf{c}-\tilde{\mathbf{c}}\|_{s} \max \left(\|\mathbf{c}\|\left\|_{s},\right\| \tilde{\mathbf{c}} \|_{s}\right)^{2}
\end{aligned}
$$


with a constant $C$ which depends on $d, s$, and $V$ but is independent of $\varepsilon$. The same estimates hold for $\hat{\mathbf{c}}, \hat{\tilde{\mathbf{c}}}, \hat{\mathbf{F}}$, and $\|\cdot\| \|_{\frac{d+1}{2}}$ instead of $\mathbf{c}, \tilde{\mathbf{c}}, \mathbf{F}$, and $\|\cdot \cdot\|_{s}$, respectively.

Proof. The estimate (12a) follows from the definition of the set $\mathcal{R}_{\varepsilon}$ of near-resonant indices. For the proof of $(12 b)$ we first note that for $\mathbf{k}^{1}+\mathbf{k}^{2}-\mathbf{k}^{3}=\mathbf{k}$

$$
\left[\left[\mathbf{k}^{1}\right]\right]+\left[\left[\mathbf{k}^{2}\right]\right]+\left[\left[\mathbf{k}^{3}\right]\right] \geq \max ([[\mathbf{k}]], 2)+1
$$

since $\left[\left[\mathbf{k}^{1}\right]\right]+\left[\left[\mathbf{k}^{2}\right]\right]+\left[\left[\mathbf{k}^{3}\right]\right] \geq \frac{1}{2}\left(\left\|\mathbf{k}^{1}\right\|+\left\|\mathbf{k}^{2}\right\|+\left\|\mathbf{k}^{3}\right\|+3\right) \geq \frac{1}{2}(\|\mathbf{k}\|+1)+1$ and $\left[\left[\mathbf{k}^{1}\right]\right]+\left[\left[\mathbf{k}^{2}\right]\right]+\left[\left[\mathbf{k}^{3}\right]\right] \geq 3$. Using this estimate we get

$$
\begin{aligned}
\|\mathbf{F}(\mathbf{c})\|_{s}^{2} & =\sum_{j \in \mathbb{Z}^{d}}\left|\omega_{j}\right|^{s}\left(\sum_{\mathbf{k}} \varepsilon^{-\max ([[\mathbf{k}]], 2)}\left|\sum_{\mathbf{k}^{1}+\mathbf{k}^{2}-\mathbf{k}^{3}=\mathbf{k}} \mathcal{F}_{j}\left(\varepsilon^{\left[\left[\mathbf{k}^{1}\right]\right]+\left[\left[\mathbf{k}^{2}\right]\right]+\left[\left[\mathbf{k}^{3}\right]\right]} c^{\mathbf{k}^{1}} c^{\mathbf{k}^{2} \overline{\mathbf{k}^{3}}}\right)\right|\right)^{2} \\
& \leq \varepsilon^{2} \sum_{j \in \mathbb{Z}^{d}}\left|\omega_{j}\right|^{s}\left(\sum_{\mathbf{k}^{1}, \mathbf{k}^{2}, \mathbf{k}^{3}}\left|\mathcal{F}_{j}\left(c^{\mathbf{k}^{1}} c^{\mathbf{k}^{2} \overline{\mathbf{k}^{3}}}\right)\right|\right)^{2}=\varepsilon^{2}\left\|\sum_{\mathbf{k}^{1}, \mathbf{k}^{2}, \mathbf{k}^{3}}\left\{c^{\mathbf{k}^{1}} c^{\mathbf{k}^{2} \overline{k^{3}}}\right\}\right\|_{s}^{2} .
\end{aligned}
$$

Using (11) from Lemma 1 we obtain (12b). The same calculation is true for $\mathbf{c}, \mathbf{F}$, and $\mid\|\cdot\| \|_{s}$ replaced by $\hat{\mathbf{c}}, \hat{\mathbf{F}}$, and $\|\mid \cdot\|_{\frac{d+1}{2}}$, respectively.

For the last inequality $(12 \mathrm{c})$ we note that $a_{1} \cdots a_{n}-b_{1} \cdots b_{n}=\sum_{j=1}^{n} 2^{-j}\left(a_{1}+b_{1}\right) \cdots\left(a_{j-1}+\right.$ $\left.b_{j-1}\right)\left(a_{j}-b_{j}\right)\left(a_{j+1} \cdots a_{n}+b_{j+1} \cdots b_{n}\right)$ (used for $n=3$ ). A calculation as above thus yields the result on $\|\mid \mathbf{F}(\mathbf{c})-\mathbf{F}(\tilde{\mathbf{c}})\| \|_{s}$ and $\|\hat{\mathbf{F}}(\hat{\mathbf{c}})-\hat{\mathbf{F}}(\hat{\tilde{\mathbf{c}}})\|_{\frac{d+1}{2}}$.

\subsection{Size of the iterated modulation functions}

We have

$$
\| \mid \mathbf{a}(0)]^{n+1} \mid\left\|_{s}=\left(\sum_{j \in \mathbb{Z}^{d}}\left|\omega_{j}\right|^{s}\left|\left[a_{j}^{\langle j\rangle}(0)\right]^{n+1}\right|^{2}\right)^{\frac{1}{2}} \leq \varepsilon^{-1}\right\| u(0)\left\|_{s}+\varepsilon\right\|\left\|[\mathbf{b}(0)]^{n}\right\| \|_{s} .
$$

Using Lemma 2 we get for $0 \leq \varepsilon t=\tau \leq 1$ and $l \geq 0$

$$
\begin{aligned}
\left\|\left[\mathbf{b}^{(l)}\right]^{n+1}\right\| \|_{s} & \leq \varepsilon^{\frac{1}{2}}\left\|\left[\mathbf{b}^{(l+1)}\right]^{n}\right\|\left\|_{s}+\varepsilon^{-\frac{1}{2}}\right\| \mathbf{F}\left([\mathbf{c}]^{n}\right)^{(l)}\|\|_{s}, \\
\left\|[\mathbf{a}]^{n+1}\right\| \|_{s} & \leq\left\|[\mathbf{a}(0)]^{n+1}\right\|\left\|_{s}+\sup _{0 \leq \tau \leq 1}\right\|[\dot{\mathbf{a}}(\tau)]^{n+1} \|_{s}, \\
\left\|\left[\mathbf{a}^{(l+1)}\right]^{n+1}\right\| \|_{s} & \leq\left\|\mathbf{F}\left([\mathbf{c}]^{n}\right)^{(l+1)}\right\|_{s}
\end{aligned}
$$

where $^{(l)}$ denotes the $l$-th derivative with respect to $\tau=\varepsilon t$. With

$$
\begin{aligned}
& \alpha_{n}=\max _{l=0, \ldots, 1+2 L-n} \sup _{0 \leq \tau \leq 1}\left\|\left[\mathbf{a}^{(l)}(\tau)\right]^{n}\right\| \|_{s}, \\
& \beta_{n}=\max _{l=0, \ldots, 1+2 L-n} \sup _{0 \leq \tau \leq 1}\|\|\left[\mathbf{b}^{(l)}(\tau)\right]^{n} \|_{s}
\end{aligned}
$$

this implies for $n=0, \ldots, L-1$, using again Lemma 2 and the smallness of the initial data, $\alpha_{n+1} \leq 1+\varepsilon \beta_{n}+C \varepsilon\left(\alpha_{n}+\beta_{n}\right)^{3}$ and $\beta_{n+1} \leq \varepsilon^{\frac{1}{2}} \beta_{n}+C \varepsilon^{\frac{1}{2}}\left(\alpha_{n}+\beta_{n}\right)^{3}$ where the constants depend on $d, L, n, s$, and $V$ but not on $\varepsilon$. The dependence on $n$ is due to the estimates of derivatives of $\mathbf{F}$ with the product rule. Using $\alpha_{0}=\left\|[\mathbf{a}(0)]^{0}\right\|_{s}=\varepsilon^{-1}\|u(0)\|_{s} \leq 1$ and $\beta_{0}=0$ we get for $n=0, \ldots, L$

$$
\alpha_{n} \leq C, \quad \beta_{n} \leq C \varepsilon^{\frac{1}{2}}
$$

with a constant $C$ which depends on $d, L, n, s$, and $V$ but not on $\varepsilon$. With these estimates we now prove the estimate $(9 \mathrm{~b})$ of Proposition 3 for $\tilde{u}=[\tilde{u}]^{L}=\sum_{\mathbf{k}}\left[z^{\mathbf{k}}\right]^{L} e^{-i(\mathbf{k} \cdot \boldsymbol{\omega}) t}$.

$$
\|\tilde{u}\|_{s}^{2}=\sum_{j \in \mathbb{Z}^{d}}\left|\omega_{j}\right|^{s}\left|\sum_{\mathbf{k}}\left[z_{j}^{\mathbf{k}}(\varepsilon t)\right]^{L} e^{-i(\mathbf{k} \cdot \boldsymbol{\omega}) t}\right|^{2} \leq \varepsilon^{2} \sum_{j \in \mathbb{Z}^{d}}\left|\omega_{j}\right|^{s}\left(\sum_{\mathbf{k}}\left|\left[c_{j}^{\mathbf{k}}(\varepsilon t)\right]^{L}\right|\right)^{2}=\left.\varepsilon^{2}\left\|[\mathbf{c}]^{L}\right\|\right|_{s} ^{2} .
$$


We now turn to the size of the variables $\hat{\mathbf{a}}$ and $\hat{\mathbf{b}}$ in the second rescaling and set

$$
\begin{aligned}
& \hat{\alpha}_{n}=\max _{l=0, \ldots, 1+2 L-n} \sup _{0 \leq \tau \leq 1}\left\|\left[\hat{\mathbf{a}}^{(l)}(\tau)\right]^{n}\right\| \|_{\frac{d+1}{2}}, \\
& \hat{\beta}_{n}=\max _{l=0, \ldots, 1+2 L-n} \sup _{0 \leq \tau \leq 1}\left\|\left[\hat{\mathbf{b}}^{(l)}(\tau)\right]^{n}\right\|_{\frac{d+1}{2}} .
\end{aligned}
$$

The relation $\|\hat{\mathbf{a}}\|_{\frac{d+1}{2}}=\|\mathbf{a}\|_{s}$ yields $\hat{\alpha}_{n}=\alpha_{n}$. For $\hat{\beta}_{n}$ we get the same estimate as for $\beta_{n}$ and so for $n=0, \ldots, L$

$$
\hat{\alpha}_{n} \leq C, \quad \hat{\beta}_{n} \leq C \varepsilon^{\frac{1}{2}}
$$

with a constant $C$ which depends on $d, L, n, s$, and $V$ but not on $\varepsilon$. Together with (14) this yields for $\mathbf{z}=[\mathbf{z}]^{L}$ the estimates (9c) of Proposition 3.

\subsection{Defect of the iterated modulation functions}

After $n$ steps the defect in the modulation system (7a) is (with $j=j(\mathbf{k})$ )

$$
\left[d_{j}^{\mathbf{k}}\right]^{n}=\left[i \varepsilon \dot{z}_{j}^{\mathbf{k}}+\left(\mathbf{k} \cdot \boldsymbol{\omega}-\omega_{j}\right) z_{j}^{\mathbf{k}}-\sum_{\mathbf{k}^{1}+\mathbf{k}^{2}-\mathbf{k}^{3}=\mathbf{k}} \mathcal{F}_{j}\left(z^{\mathbf{k}^{1}} z^{\mathbf{k}^{2}} \overline{z^{\mathbf{k}^{3}}}\right)\right]^{n} .
$$

This has to be considered for $\|\mathbf{k}\| \leq 3 K$ where we set $\left[z^{\mathbf{k}}\right]^{n}=0$ for $\|\mathbf{k}\|>K$ and all $n$. We decompose the defect in (7a) as $d_{j}^{\mathbf{k}}=e_{j}^{\mathbf{k}}+f_{j}^{\mathbf{k}}+g_{j}^{\mathbf{k}}$ with $e_{j}^{\mathbf{k}}=0$ for $(j, \mathbf{k}) \notin \mathcal{S}_{\varepsilon}$ and $\mathbf{k} \neq\langle j\rangle, f_{j}^{\mathbf{k}}=0$ for $(j, \mathbf{k}) \notin \mathcal{R}_{\varepsilon}$ and $g_{j}^{\mathbf{k}}=0$ for $\|\mathbf{k}\| \leq K$. The defect in equation (7b) for the initial condition reads

$$
\left[\tilde{d}_{j}^{\langle j\rangle}\right]^{n}=u_{j}(0)-\left[\sum_{\mathbf{k}} z_{j}^{\mathbf{k}}(0)\right]^{n} .
$$

To estimate $\mathbf{f}$ we make use of the non-resonance condition (5). With Lemma 2 and the estimates (15) we get for $n=0, \ldots, L$

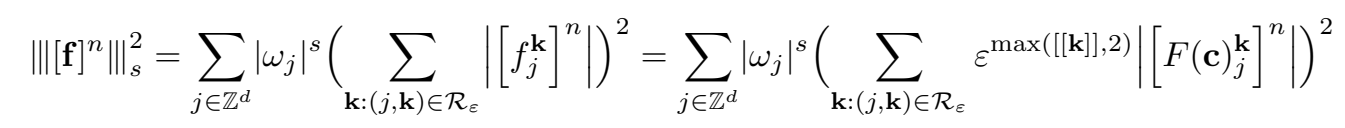

$$
\begin{aligned}
& =\sum_{j \in \mathbb{Z}^{d}}\left|\omega_{j}\right|^{\frac{d+1}{2}}\left(\sum_{\mathbf{k}:(j, \mathbf{k}) \in \mathcal{R}_{\varepsilon}} \frac{\left|\omega_{j}\right|^{\frac{2 s-d-1}{4}} \varepsilon^{\max ([[\mathbf{k}]], 2)}}{\left|\omega^{\frac{2 s-d-1}{4}|\mathbf{k}|}\right|}\left|\left[\hat{F}(\hat{\mathbf{c}})_{j}^{\mathbf{k}}\right]^{n}\right|\right)^{2} \\
& \leq\left\|[\hat{\mathbf{F}}(\hat{\mathbf{c}})]^{n}\right\|_{\frac{d+1}{2}}^{2} \sup _{(j, \mathbf{k}) \in \mathcal{R}_{\varepsilon}}\left(\frac{\left|\omega_{j}\right|^{\frac{2 s-d-1}{4}}}{\left|\boldsymbol{\omega}^{\frac{2 s-d-1}{4}|\mathbf{k}|}\right|} \varepsilon^{[[\mathbf{k}]]}\right)^{2} \leq\left(C \varepsilon^{N+3}\right)^{2} .
\end{aligned}
$$

with a constant which depends on $C_{0}, d, N, n, s$, and $V$ but not on $\varepsilon$. With the same arguments as in the proof of Lemma 2 we obtain for $\mathbf{g}$ using in addition (14)

$$
\begin{aligned}
\left\|\sum_{K<\|\mathbf{k}\| \leq 3 K}\left\{\left[g^{\mathbf{k}}\right]^{n}\right\}\right\|_{s} & =\sum_{K<\|\mathbf{k}\| \leq 3 K} \varepsilon^{[[\mathbf{k}]]}\left\{\varepsilon^{-[[\mathbf{k}]]} \sum_{\mathbf{k}^{1}+\mathbf{k}^{2}-\mathbf{k}^{3}=\mathbf{k}} \varepsilon^{\left[\left[\mathbf{k}^{1}\right]\right]+\left[\left[\mathbf{k}^{2}\right]\right]+\left[\left[\mathbf{k}^{3}\right]\right]} c^{\mathbf{k}^{1}} c^{\mathbf{k}^{2}} c^{\mathbf{k}^{3}}\right\} \|_{s} \\
& \leq C \varepsilon^{\frac{1}{2}(K+2)} \varepsilon=C \varepsilon^{N+3}
\end{aligned}
$$

with a constant which depends on $d, N, n, s$, and $V$ but not on $\varepsilon$.

The remainder of this subsection is devoted to the analysis of $\mathbf{e}$,

We have

$$
\left[e_{j}^{\mathbf{k}}\right]^{n}= \begin{cases}\varepsilon^{[[k]]}\left(\left[(\boldsymbol{\Omega} \mathbf{b})_{j}^{\mathbf{k}}\right]^{n}-\left[(\boldsymbol{\Omega} \mathbf{b})_{j}^{\mathbf{k}}\right]^{n+1}\right), & (j, \mathbf{k}) \in \mathcal{S}_{\varepsilon}, \\ i \varepsilon^{\frac{3}{2}}\left(\left[(\boldsymbol{\Omega} \dot{\mathbf{a}})_{j}^{\mathbf{k}}\right]^{n}-\left[(\boldsymbol{\Omega} \mathbf{a})_{j}^{\mathbf{k}}\right]^{n+1}\right), & \mathbf{k}=\langle j\rangle .\end{cases}
$$

$$
\begin{aligned}
\boldsymbol{\Omega}\left([\mathbf{b}]^{n}-[\mathbf{b}]^{n+1}\right)_{j}^{\mathbf{k}} & =-i \varepsilon\left([\dot{\mathbf{b}}]^{n-1}-[\dot{\mathbf{b}}]^{n}\right)_{j}^{\mathbf{k}}+\left(\mathbf{F}\left([\mathbf{c}]^{n-1}\right)-\mathbf{F}\left([\mathbf{c}]^{n}\right)\right)_{j}^{\mathbf{k}} \quad \text { for }(j, \mathbf{k}) \in \mathcal{S}_{\varepsilon}, \\
\left(\boldsymbol{\Omega}\left([\mathbf{a}(0)]^{n}-[\mathbf{a}(0)]^{n+1}\right)\right)_{j}^{\langle j\rangle} & =\varepsilon^{\frac{1}{2}} \sum_{\mathbf{k} \neq\langle j\rangle} \varepsilon^{[[\mathbf{k}]]-1}\left(\left[b_{j}^{\mathbf{k}}(0)\right]^{n-1}-\left[b_{j}^{\mathbf{k}}(0)\right]^{n}\right), \\
\boldsymbol{\Omega}\left([\dot{\mathbf{a}}]^{n}-[\dot{\mathbf{a}}]^{n+1}\right)_{j}^{\langle j\rangle} & =-i \varepsilon^{\frac{1}{2}}\left(\mathbf{F}\left([\mathbf{c}]^{n-1}\right)-\mathbf{F}\left([\mathbf{c}]^{n}\right)\right)_{j}^{\langle j\rangle}
\end{aligned}
$$


In particular we have with Lemma 2

$$
\left\|\boldsymbol{\Omega}\left([\mathbf{a}(0)]^{n}-[\mathbf{a}(0)]^{n+1}\right)\right\|_{s} \leq \varepsilon\left\|\boldsymbol{\Omega}\left([\mathbf{b}(0)]^{n-1}-[\mathbf{b}(0)]^{n}\right)\right\| \|_{s} .
$$

As in the analysis of the size of the modulation functions we set

$$
\begin{aligned}
& \eta_{n}=\max _{l=0, \ldots, 2 L-n} \sup _{0 \leq \tau \leq 1}\left\|\boldsymbol{\Omega}\left(\left[\mathbf{a}^{(l)}(\tau)\right]^{n}-\left[\mathbf{a}^{(l)}(\tau)\right]^{n+1}\right)\right\| \|_{s}, \\
& \mu_{n}=\max _{l=0, \ldots, 2 L-n} \sup _{0 \leq \tau \leq 1}\left\|\boldsymbol{\Omega}\left(\left[\mathbf{b}^{(l)}(\tau)\right]^{n}-\left[\mathbf{b}^{(l)}(\tau)\right]^{n+1}\right)\right\| \|_{s} .
\end{aligned}
$$

By Lemma 2 and (14) we have $\eta_{n+1} \leq \varepsilon \mu_{n}+C \varepsilon\left(\eta_{n}+\mu_{n}\right)$ and $\mu_{n+1} \leq \varepsilon^{\frac{1}{2}} \mu_{n}+C \varepsilon^{\frac{1}{2}}\left(\eta_{n}+\mu_{n}\right)$ for $n=0, \ldots, L-1$. We remark that we gain a factor of $\varepsilon^{\frac{1}{2}}$ in each iteration step whereas in $[6$, Subsection 3.11] the variables had to be rescaled once more to gain a positive power of $\varepsilon$. The reason is that the nonlinearity in (1) is cubic and not only quadratic as in [6]. Using (14) we get for the initial values $\eta_{0} \leq \varepsilon^{\frac{1}{2}}\left(\alpha_{0}+\alpha_{1}\right) \leq C \varepsilon^{\frac{1}{2}}$ and $\mu_{0} \leq\left\|\boldsymbol{\Omega}\left([\mathbf{b}(0)]^{1}\right)\right\|\left\|_{s} \leq\right\| \mathbf{F}\left([\mathbf{c}(0)]^{0}\right)\|\|_{s} \leq C \varepsilon$. Similar estimates are true for the second rescaling with

$$
\begin{aligned}
& \hat{\eta}_{n}=\max _{l=0, \ldots, 2 L-n} \sup _{0 \leq \tau \leq 1}\left\|\boldsymbol{\Omega}\left(\left[\hat{\mathbf{a}}^{(l)}(\tau)\right]^{n}-\left[\hat{\mathbf{a}}^{(l)}(\tau)\right]^{n+1}\right)\right\|_{\frac{d+1}{2},}, \\
& \hat{\mu}_{n}=\max _{l=0, \ldots, 2 L-n} \sup _{0 \leq \tau \leq 1}\left\|\boldsymbol{\Omega}\left(\left[\hat{\mathbf{b}}^{(l)}(\tau)\right]^{n}-\left[\hat{\mathbf{b}}^{(l)}(\tau)\right]^{n+1}\right)\right\|_{\frac{d+1}{2}} .
\end{aligned}
$$

(Note that $\hat{\eta}_{n}=\eta_{n}$.) We have thus proven that for $n=0, \ldots, L$

$$
\eta_{n}, \mu_{n}, \hat{\eta}_{n}, \hat{\mu}_{n} \leq C \varepsilon^{\frac{n+1}{2}}
$$

with a constant $C$ which depends on $d, L, n, s$, and $V$ but not on $\varepsilon$. Hence with Lemma 2 $\left\|[\mathbf{e}]^{n}\right\|_{s} \leq C \varepsilon^{\frac{n+4}{2}}$ and $\left\|[\hat{\mathbf{e}}]^{n}\right\| \|_{\frac{d+1}{2}} \leq C \varepsilon^{\frac{n+4}{2}}$ which yields together with (16) the estimates (9d) of Proposition 3. (18)

For the defect $\tilde{\mathbf{d}}$ in the initial conditions we get using the iteration for $\left[z_{j}^{\langle j\rangle}(0)\right]^{n}$, Lemma 2, and

$$
\begin{aligned}
\left\|[\tilde{\mathbf{d}}]^{n}\right\| \|_{s}^{2} & =\sum_{j \in \mathbb{Z}^{d}}\left|\omega_{j}\right|^{s}\left|u_{j}(0)-\sum_{\mathbf{k}}\left[z_{j}^{\mathbf{k}}(0)\right]^{n}\right|^{2}=\sum_{j \in \mathbb{Z}^{d}}\left|\omega_{j}\right|^{s}\left|\sum_{\mathbf{k} \neq\langle j\rangle}\left(\left[z_{j}^{\mathbf{k}}(0)\right]^{n-1}-\left[z_{j}^{\mathbf{k}}(0)\right]^{n}\right)\right|^{2} \\
& \leq\left\|\boldsymbol{\Omega}^{-1}\left([\mathbf{e}(0)]^{n-1}\right)\right\|_{s}^{2} \leq\left(C \varepsilon^{-\frac{1}{2}} \varepsilon^{\frac{n+3}{2}}\right)^{2}
\end{aligned}
$$

for $n=1, \ldots, L$.

\subsection{Error}

We now turn to the proof of the estimate (9a) of Proposition 3. We write $\tilde{u}=[\tilde{u}]^{L}=\sum_{\mathbf{k}}\left[z^{\mathbf{k}}\right]^{L} e^{-i(\mathbf{k} \cdot \boldsymbol{\omega}) t}$ and $\mathbf{z}=[\mathbf{z}]^{L}$, and estimate the error $\tilde{u}-u$ where $u$ is the exact solution of the nonlinear Schrödinger equation (1).

\subsubsection{Size of the solution}

We first determine the size of the solution $u$ of (1). This solution satisfies

$$
\left(u_{j}\right)_{t}=-i \omega_{j} u_{j}-i \mathcal{F}_{j}\left(|u|^{2} u\right)
$$

and the variation-of-constants formula yields

$$
u_{j}(t)=e^{-i \omega_{j} t} u_{j}(0)-\int_{0}^{t} e^{-i \omega_{j}(t-\theta)} i \mathcal{F}_{j}\left(|u(\cdot, \theta)|^{2} u(\cdot, \theta)\right) d \theta .
$$

While $\|u(\cdot, t)\|_{s} \leq 2 \varepsilon$ we have using Lemma 1

$$
\|u(\cdot, t)\|_{s} \leq\|u(\cdot, 0)\|_{s}+\int_{0}^{t} C^{2}\|u(\cdot, \theta)\|_{s}^{3} d \theta \leq\|u(\cdot, 0)\|_{s}+\int_{0}^{t} 4 C^{2} \varepsilon^{2}\|u(\cdot, \theta)\|_{s} d \theta .
$$

The Gronwall inequality yields $\|u(\cdot, t)\|_{s} \leq\|u(\cdot, 0)\|_{s} e^{4 C^{2} \varepsilon^{2} t}$. So we have for $\varepsilon \leq \log (2) /\left(4 C^{2}\right)$

$$
\|u(\cdot, t)\|_{s} \leq 2 \varepsilon \text { for } 0 \leq t \leq \varepsilon^{-1} .
$$




\subsubsection{Error on $\left[0, \varepsilon^{-1}\right]$}

In $t=0$ we have by (19)

$$
\|\tilde{u}(\cdot, 0)-u(\cdot, 0)\|_{s} \leq\|\|[\tilde{\mathbf{d}}]^{L} \|_{s} \leq C \varepsilon^{N+2}
$$

with a constant which depends on $d, N, s$, and $V$ but not on $\varepsilon$.

On $\left[0, \varepsilon^{-1}\right]$ we have

$$
\left(\tilde{u}_{j}-u_{j}\right)_{t}+i \omega_{j}\left(\tilde{u}_{j}-u_{j}\right)+i \mathcal{F}_{j}\left(|\tilde{u}|^{2} \tilde{u}-|u|^{2} u\right)=-i \sum_{\|\mathbf{k}\| \leq 3 K}\left[d_{j}^{\mathbf{k}}\right]^{L} e^{-i(\mathbf{k} \cdot \boldsymbol{\omega}) t} e^{i(j \cdot x)}=: \delta_{j} .
$$

The variation-of-constants formula yields

$$
\begin{aligned}
\tilde{u}_{j}(t)-u_{j}(t)= & e^{-i \omega_{j} t}\left(\tilde{u}_{j}(0)-u_{j}(0)\right) \\
& \quad-\int_{0}^{t} e^{-i \omega_{j}(t-\theta)}\left(i \mathcal{F}_{j}\left(|\tilde{u}(\cdot, \theta)|^{2} \tilde{u}(\cdot, \theta)-|u(\cdot, \theta)|^{2} u(\cdot, \theta)\right)-\delta_{j}(\cdot, \theta)\right) d \theta .
\end{aligned}
$$

The integrand is estimated for $0 \leq t \leq \varepsilon^{-1}$ with (16), (17), (18), and

$$
2\left\||\tilde{u}|^{2} \tilde{u}-|u|^{2} u\right\|_{s} \leq\left\|\overline{(\tilde{u}-u)} \tilde{u}^{2}\right\|_{s}+\left\|\overline{(\tilde{u}-u)} u^{2}\right\|_{s}+\left\||\tilde{u}+u|^{2}(\tilde{u}-u)\right\|_{s} \leq C \varepsilon^{2}\|\tilde{u}-u\|_{s}
$$

by Lemma $1,(20)$, and the inequality $(9 \mathrm{~b})$. We thus obtain

$$
\|\tilde{u}(\cdot, t)-u(\cdot, t)\|_{s} \leq\|\tilde{u}(\cdot, 0)-u(\cdot, 0)\|_{s}+\int_{0}^{t} C \varepsilon^{2}\|\tilde{u}(\cdot, \theta)-u(\cdot, \theta)\|_{s} d \theta+t C \varepsilon^{N+3}
$$

with a constant which depends on $C_{0}, d, N, s$, and $V$ but not on $\varepsilon$. Together with (21) and the Gronwall inequality this yields the estimate (9a) in Proposition 3 completing the proof of this proposition.

\subsection{Interface between modulated Fourier expansions}

So far, we have constructed an approximate solution $\mathbf{z}=[\mathbf{z}]^{L}$ of the modulation system (7) for $0 \leq \varepsilon t \leq 1$. With the same method we can construct an approximate solution $\tilde{\mathbf{z}}$ of the modulation system (7) for $1 \leq \varepsilon t \leq 2$ taking $u\left(\cdot, \varepsilon^{-1}\right)$ as initial value. Hence, equation (7b) becomes

$$
\sum_{\mathbf{k}} \tilde{z}_{j}^{\mathbf{k}}(1) e^{-i(\mathbf{k} \cdot \boldsymbol{\omega}) \varepsilon^{-1}}=u_{j}\left(\varepsilon^{-1}\right)
$$

The following proposition bounds the difference of $\mathbf{z}(1)$ and $\tilde{\mathbf{z}}(1)$.

Proposition 4. Assume $\left\|u\left(\cdot, \varepsilon^{-1}\right)\right\|_{s} \leq \varepsilon$. We have

$$
\|\hat{\mathbf{z}}(1)-\hat{\tilde{\mathbf{z}}}(1)\|_{\frac{d+1}{2}} \leq C \varepsilon^{N+2}
$$

with constants depending on $d, N, s$, and $V$ but not on $\varepsilon$.

Proof. As in the previous sections we use the notations $\tilde{\mathbf{a}}, \tilde{\mathbf{b}}, \hat{\tilde{\mathbf{a}}}$, and $\hat{\tilde{\mathbf{b}}}$, cf. Subsection 3.4. The iteration for $\tilde{a}_{j}^{\langle j\rangle}(1)$ reads

$$
\left[\tilde{a}_{j}^{\langle j\rangle}(1)\right]^{n+1}=\varepsilon^{-1} u_{j}\left(\varepsilon^{-1}\right) e^{i \omega_{j} \varepsilon^{-1}}-\left[\sum_{\mathbf{k} \neq\langle j\rangle} \varepsilon^{[[\mathbf{k}]]-1} \tilde{b}_{j}^{\mathbf{k}}(1) e^{-i\left(\mathbf{k} \cdot \boldsymbol{\omega}-\omega_{j}\right) \varepsilon^{-1}}\right]^{n} .
$$

This yields

$$
\begin{aligned}
\| \mathbf{a}(1)- & {[\tilde{\mathbf{a}}(1)]^{n+1}\|\|_{s} \leq\left(\sum_{j \in \mathbb{Z}^{d}}\left|\omega_{j}\right|^{s}\left|\sum_{\mathbf{k} \neq\langle j\rangle} \varepsilon^{[[\mathbf{k}]]-1}\left(\left[\tilde{b}_{j}^{\mathbf{k}}(1)\right]^{n}-b_{j}^{\mathbf{k}}(1)\right) e^{i\left(\omega_{j}-\mathbf{k} \cdot \boldsymbol{\omega}\right) \varepsilon^{-1}}\right|^{2}\right)^{\frac{1}{2}} } \\
& +\left(\sum_{j \in \mathbb{Z}^{d}}\left|\omega_{j}\right|^{s}\left|a_{j}^{\langle j\rangle}(1)+\sum_{\mathbf{k} \neq\langle j\rangle} \varepsilon^{[[\mathbf{k}]]-1} b_{j}^{\mathbf{k}}(1) e^{i\left(\omega_{j}-\mathbf{k} \cdot \boldsymbol{\omega}\right) \varepsilon^{-1}}-\varepsilon^{-1} u_{j}\left(\varepsilon^{-1}\right) e^{i \omega_{j} \varepsilon^{-1}}\right|^{2}\right)^{\frac{1}{2}} \\
\leq & \varepsilon\left\|[\tilde{\mathbf{b}}(1)]^{n}-\mathbf{b}(1)\right\|_{s}+\varepsilon^{-1}\left\|\tilde{u}\left(\cdot, \varepsilon^{-1}\right)-u\left(\cdot, \varepsilon^{-1}\right)\right\|_{s} .
\end{aligned}
$$


In addition we have

$$
\begin{gathered}
\left(\mathbf{b}(1)-[\tilde{\mathbf{b}}(1)]^{n+1}\right)_{j}^{\mathbf{k}}=\left(\boldsymbol{\Omega}^{-1} \boldsymbol{\Omega}\left(\mathbf{b}(1)-[\mathbf{b}(1)]^{L+1}\right)\right)_{j}^{\mathbf{k}}-i \varepsilon\left(\boldsymbol{\Omega}^{-1}\left(\dot{\mathbf{b}}(1)-[\dot{\tilde{\mathbf{b}}}(1)]^{n}\right)\right)_{j}^{\mathbf{k}} \\
+\left(\boldsymbol{\Omega}^{-1}\left(\mathbf{F}(\mathbf{c}(1))-\mathbf{F}\left([\tilde{\mathbf{c}}(1)]^{n}\right)\right)\right)_{j}^{\mathbf{k}} \text { for }(j, \mathbf{k}) \in \mathcal{S}_{\varepsilon}, \\
\left(\dot{\mathbf{a}}(1)-[\dot{\tilde{\mathbf{a}}}(1)]^{n+1}\right)_{j}^{\langle j\rangle}=\left(\dot{\mathbf{a}}(1)-[\dot{\mathbf{a}}(1)]^{L+1}\right)_{j}^{\langle j\rangle}-i\left(\mathbf{F}(\mathbf{c}(1))-\mathbf{F}\left([\tilde{\mathbf{c}}(1)]^{n}\right)\right)_{j}^{\langle j\rangle}
\end{gathered}
$$

For

$$
\begin{aligned}
\rho_{n} & =\max _{l=0, \ldots, 1+(L-n)}\left\|\mathbf{a}^{(l)}(1)-\left[\tilde{\mathbf{a}}^{(l)}(1)\right]^{n}\right\| \|_{s}, \\
\xi_{n} & =\max _{l=0, \ldots, 1+(L-n)}\left\|\mathbf{b}^{(l)}(1)-\left[\tilde{\mathbf{b}}^{(l)}(1)\right]^{n}\right\|_{s}
\end{aligned}
$$

we note $\rho_{0} \leq \alpha_{L}+\varepsilon^{-1}\left\|u\left(\varepsilon^{-1}\right)\right\|_{s} \leq C$ and $\xi_{0} \leq \beta_{L} \leq C \varepsilon^{\frac{1}{2}}$. For $n=0, \ldots, L-1$ we have with Lemma 2, (14), and (9a) $\rho_{n+1} \leq \varepsilon \xi_{n}+C \varepsilon^{-1} \varepsilon^{N+2}+\varepsilon^{-\frac{1}{2}} \eta_{L}+C \varepsilon\left(\rho_{n}+\xi_{n}\right)$ and $\xi_{n+1} \leq$ $\varepsilon^{-\frac{1}{2}} \mu_{L}+C \varepsilon^{\frac{1}{2}} \xi_{n}+C \varepsilon^{\frac{1}{2}}\left(\rho_{n}+\xi_{n}\right)$, and thus $\rho_{n}, \xi_{n} \leq C\left(\varepsilon^{N+1}+\varepsilon^{\frac{L}{2}}+\varepsilon^{\frac{n}{2}}\right)$ by (18) with a constant which depends on $d, L, n, s$, and $V$ but not on $\varepsilon$. The same procedure can be done for the second rescaling. This proves the estimates of the proposition.

\section{Conservation properties}

The modulation system (7a) has invariants close to the actions as we discuss now. Let $\mathbf{z}$ be the functions of Proposition 3.

\subsection{Almost-invariants of the modulation system}

Let

$$
U(\mathbf{z})=\sum_{\mathbf{k}^{1}+\mathbf{k}^{2}-\mathbf{k}^{3}-\mathbf{k}^{4}=\mathbf{0}} \frac{1}{(2 \pi)^{d}} \int_{\mathbb{T}^{d}} z^{\mathbf{k}^{1}} z^{\mathbf{k}^{2}} z^{\mathbf{k}^{3} z^{\mathbf{k}^{4}}} d x .
$$

The transformation $z^{\mathbf{k}} \mapsto e^{i(\mathbf{k} \cdot \boldsymbol{\mu}) \theta} z^{\mathbf{k}}$ for real sequences $\boldsymbol{\mu}=\left(\mu_{l}\right)_{l \in \mathbb{Z}^{d}}$ and $\theta \in \mathbb{R}$ leaves $U$ invariant since the sum is over $\mathbf{k}^{1}+\mathbf{k}^{2}-\mathbf{k}^{3}-\mathbf{k}^{4}=\mathbf{0}$. Hence, we have

$$
\begin{aligned}
0= & \left.\frac{d}{d \theta}\right|_{\theta=0} U\left(\left(e^{i(\mathbf{k} \cdot \boldsymbol{\mu}) \theta} z^{\mathbf{k}}\right)_{\mathbf{k}}\right)=\sum_{\mathbf{k}} \frac{1}{(2 \pi)^{d}} \int_{\mathbb{T}^{d}} 2 i(\mathbf{k} \cdot \boldsymbol{\mu}) z^{\mathbf{k}} \sum_{\mathbf{k}^{2}-\mathbf{k}^{3}-\mathbf{k}^{4}=-\mathbf{k}} z^{\mathbf{k}^{2}} \overline{z^{\mathbf{k}^{3}} z^{\mathbf{k}^{4}}} d x \\
& +\sum_{\mathbf{k}} \frac{1}{(2 \pi)^{d}} \int_{\mathbb{T}^{d}} 2(-i)(\mathbf{k} \cdot \boldsymbol{\mu}) \overline{z^{\mathbf{k}}} \sum_{\mathbf{k}^{1}+\mathbf{k}^{2}-\mathbf{k}^{3}=\mathbf{k}} z^{\mathbf{k}^{1}} z^{\mathbf{k}^{2}} \overline{z^{\mathbf{k}^{3}}} d x \\
=- & 4 \operatorname{Re}\left(\sum_{\mathbf{k}} i(\mathbf{k} \cdot \boldsymbol{\mu}) \sum_{j \in \mathbb{Z}^{d}} \overline{z_{j}^{\mathbf{k}}} \mathcal{F}_{j}\left(\sum_{\mathbf{k}^{1}+\mathbf{k}^{2}-\mathbf{k}^{3}=\mathbf{k}} z^{\mathbf{k}^{1}} z^{\mathbf{k}^{2}} \overline{z^{\mathbf{k}^{3}}}\right)\right)
\end{aligned}
$$

and with (7a) and $2 \operatorname{Re}\left(\overline{z_{j}^{\mathbf{k}}} \varepsilon \dot{z}_{j}^{\mathbf{k}}\right)=\frac{d}{d t}\left|z_{j}^{\mathbf{k}}\right|^{2}$ we obtain

$$
0=2 \frac{d}{d t} \sum_{\mathbf{k}}(\mathbf{k} \cdot \boldsymbol{\mu})\left|z_{j(\mathbf{k})}^{\mathbf{k}}\right|^{2}+4 \operatorname{Re}\left(\sum_{\mathbf{k}} i(\mathbf{k} \cdot \boldsymbol{\mu}) \overline{z_{j(\mathbf{k})}^{\mathbf{k}}} d_{j(\mathbf{k})}^{\mathbf{k}}\right) .
$$

Hence

$$
\mathcal{I}_{\boldsymbol{\mu}}(\mathbf{z}(\cdot, \varepsilon t))=\frac{1}{2} \sum_{\mathbf{k}}(\mathbf{k} \cdot \boldsymbol{\mu})\left|z_{j(\mathbf{k})}^{\mathbf{k}}(\varepsilon t)\right|^{2}
$$

is an almost-invariant of the modulation system (7a). To quantify the term "almost" we need the following lemma.

Lemma 3. Let $\mathbf{z}$ and $\mathbf{r}=\mathbf{p}+\mathbf{q}$ with $z_{j}^{\mathbf{k}}=p_{j}^{\mathbf{k}}=0$ for $j \neq j(\mathbf{k})$ or $(j, \mathbf{k}) \in \mathcal{R}_{\varepsilon}$ and $q_{j}^{\mathbf{k}}=0$ for $(j, \mathbf{k}) \notin \mathcal{R}_{\varepsilon}$. The following estimate holds for $s \geq d+1$ with a constant $C$ which depends only on $d, K, s$, and $V$.

$$
\sum_{l \in \mathbb{Z}^{d}}\left|\omega_{l}\right|^{s} \sum_{\mathbf{k}}\left|k_{l}\left\|z_{j(\mathbf{k})}^{\mathbf{k}}|| r_{j(\mathbf{k})}^{\mathbf{k}}|\leq C\|\hat{\mathbf{z}}\||_{\frac{d+1}{2}}\right\| \hat{\mathbf{p}} \|\right|_{\frac{d+1}{2}} .
$$


In addition we have for $\mathbf{q}=\mathbf{0}$

$$
\sum_{l \in \mathbb{Z}^{d}}\left|\omega_{l}\right|^{s}\left|\mathcal{I}_{\langle l\rangle}(\mathbf{z})-\mathcal{I}_{\langle l\rangle}(\mathbf{r})\right| \leq C\|\hat{\mathbf{z}}-\hat{\mathbf{p}}\|\left\|_{\frac{d+1}{2}}\right\| \hat{\mathbf{z}}+\hat{\mathbf{p}} \|_{\frac{d+1}{2}}
$$

Proof. Since $z_{j}^{\mathbf{k}}=0$ for $(j, \mathbf{k}) \in \mathcal{R}_{\varepsilon}$ we have

$$
\sum_{l \in \mathbb{Z}^{d}}\left|\omega_{l}\right|^{s} \sum_{\mathbf{k}}\left|k_{l}\right|\left|z_{j(\mathbf{k})}^{\mathbf{k}}\right|\left|r_{j(\mathbf{k})}^{\mathbf{k}}\right|=\left.\sum_{\mathbf{k}=\langle j\rangle}\left|\operatorname{or}_{(j, \mathbf{k}) \in \mathcal{S}_{\varepsilon}}\right| \omega_{j}\right|^{\frac{d+1}{2}} \frac{\sum_{l \in \mathbb{Z}^{d}}\left|k_{l}\right|\left|\omega_{l}\right|^{s}}{\left|\boldsymbol{\omega}^{\left(s-\frac{d+1}{2}\right)|\mathbf{k}|}\right|\left|\omega_{j}\right|^{\frac{d+1}{2}}}\left|\hat{z}_{j}^{\mathbf{k}}\right|\left|\hat{p}_{j}^{\mathbf{k}}\right|
$$

where $\mathcal{S}_{\varepsilon}=\left\{(j, \mathbf{k}): j=j(\mathbf{k}), \mathbf{k} \neq\langle j\rangle,(j, \mathbf{k}) \notin \mathcal{R}_{\varepsilon},\|\mathbf{k}\| \leq K\right\}$. We now bound

$$
\sup _{\mathbf{k}=\langle j\rangle \text { or }(j, \mathbf{k}) \in \mathcal{S}_{\varepsilon}} \frac{\sum_{l \in \mathbb{Z}^{d}}\left|k_{l}\right|\left|\omega_{l}\right|^{s}}{\left|\boldsymbol{\omega}^{\left(s-\frac{d+1}{2}\right)|\mathbf{k}|}\right|\left|\omega_{j}\right|^{\frac{d+1}{2}}} .
$$

For $\mathbf{k}=\langle j\rangle$ the fraction is 1 . Let now $(j, \mathbf{k})=(j(\mathbf{k}), \mathbf{k}) \in \mathcal{S}_{\varepsilon}$. We have for the nominator in (23) $\sum_{l \in \mathbb{Z}^{d}}\left|k_{l}\right|\left|\omega_{l}\right|^{s} \leq C\left|\omega_{L}\right|^{s}$ if $L$ is the index of largest norm $|\cdot|$ with $k_{L} \neq 0$. The constant $C$ depends only on $K, s$, and $V$.

For the estimation of the denominator in (23) we consider two cases. For $\mathbf{k}$ with $\left|k_{L}\right|>1$ or $k_{l} \neq 0$ for $l \neq L$ with $|l|>\frac{1}{2 K}|L|$ we have $\left|\boldsymbol{\omega}^{\left(s-\frac{d+1}{2}\right)|\mathbf{k}|}\right| \geq C\left|\omega_{L}\right|^{s-\frac{d+1}{2}}\left|\omega_{L}\right|^{\frac{d+1}{2}}$ because of $s \geq d+1$ for a constant which depends on $d, K, s$, and $V$. For the other $\mathbf{k}$ we have

$$
|j(\mathbf{k})|=\left|\sum_{l \in \mathbb{Z}^{d}} k_{l} l\right|=\left|k_{L} L+\sum_{|l| \leq \frac{1}{2 K}|L|} k_{l} l\right| \geq|L|-\frac{K-1}{2 K}|L|>\frac{1}{2}|L|
$$

and hence $\left|\boldsymbol{\omega}^{\left(s-\frac{d+1}{2}\right)|\mathbf{k}|}\right|\left|\omega_{j}\right|^{\frac{d+1}{2}} \geq C\left|\omega_{L}\right|^{s-\frac{d+1}{2}}\left|\omega_{L}\right|^{\frac{d+1}{2}}$ with a constant which depends on $d, s$, and $V$. Thus, (23) is bounded by a constant depending on $d, K, s$, and $V$.

The Cauchy-Schwarz inequality now yields the first estimate of the lemma. For the proof of the second estimate we just remark

$$
\sum_{l \in \mathbb{Z}^{d}}\left|\omega_{l}\right|^{s}\left|\mathcal{I}_{\langle l\rangle}(\mathbf{z})-\mathcal{I}_{\langle l\rangle}(\mathbf{r})\right| \leq\left.\sum_{l \in \mathbb{Z}^{d}}\left|\omega_{l}\right|^{s} \sum_{\mathbf{k}}\left|k_{l}\right||| z_{j(\mathbf{k})}^{\mathbf{k}}\right|^{2}-\left|r_{j(\mathbf{k})}^{\mathbf{k}}\right|^{2} \mid
$$

and $\left.|| z_{j}^{\mathbf{k}}\right|^{2}-\left|r_{j}^{\mathbf{k}}\right|^{2}|\leq| z_{j}^{\mathbf{k}}-r_{j}^{\mathbf{k}}|| z_{j}^{\mathbf{k}}+r_{j}^{\mathbf{k}} \mid$.

Using Lemma 3 and Proposition 3 we obtain the following lemma concerning the conservation of $\mathcal{I}_{\langle l\rangle}$ from (22).

Lemma 4. We have for $0 \leq t \leq \varepsilon^{-1}$

$$
\sum_{l \in \mathbb{Z}^{d}}\left|\omega_{l}\right|^{s}\left|\frac{d}{d t} \mathcal{I}_{\langle l\rangle}(\mathbf{z}(\cdot, \varepsilon t))\right| \leq C \varepsilon^{N+3}
$$

with a constant $C$ which depends on $C_{0}, d, N, s$, and $V$ but not on $\varepsilon$ and $t$.

\subsection{Relationship between almost-invariants and actions}

Lemma 5. We have for $0 \leq t \leq \varepsilon^{-1}$

$$
\sum_{l \in \mathbb{Z}^{d}}\left|\omega_{l}\right|^{s}\left|\mathcal{I}_{\langle l\rangle}(\mathbf{z}(\cdot, \varepsilon t))-I_{l}(u(\cdot, t), \overline{u(\cdot, t)})\right| \leq C \varepsilon^{\frac{7}{2}}
$$

with a constant $C$ which depends on $C_{0}, d, N, s$, and $V$ but not on $\varepsilon$ and $t$. 
Proof. On the one hand we have using Lemma 3 and Proposition 3

$$
\left.\left.\left.\sum_{l \in \mathbb{Z}^{d}}\left|\omega_{l}\right|^{s}\left|\mathcal{I}_{\langle l\rangle}(\mathbf{z})-\frac{1}{2}\right| z_{l}^{\langle l\rangle}\right|^{2}\left|=\frac{1}{2} \sum_{l \in \mathbb{Z}^{d}}\right| \omega_{l}\right|^{s}\left|\sum_{\mathbf{k} \neq\langle l\rangle} k_{l}\right| z_{j(\mathbf{k})}^{\mathbf{k}}\right|^{2} \mid \leq C \varepsilon^{5}
$$

where $z_{j}^{\mathbf{k}}$ is evaluated at $\varepsilon t$. On the other hand we have using the Cauchy-Schwarz inequality

$$
\left.\sum_{l \in \mathbb{Z}^{d}}\left|\omega_{l}\right|^{s}|| u_{l}\right|^{2}-\left|z_{l}^{\langle l\rangle}\right|^{2} \mid \leq\left(\|u-\tilde{u}\|_{s}+\left(\sum_{l \in \mathbb{Z}^{d}}\left|\omega_{l}\right|^{s}\left(\sum_{\mathbf{k} \neq\langle l\rangle}\left|z_{l}^{\mathbf{k}}\right|\right)^{2}\right)^{\frac{1}{2}}\right)\left(\|u\|_{s}+\left(\sum_{l \in \mathbb{Z}^{d}}\left|\omega_{l}\right|^{s}\left|z_{l}^{\langle l\rangle}\right|^{2}\right)^{\frac{1}{2}}\right)
$$

since

$$
\left.|| u_{l}\right|^{2}-\left|z_{l}^{\langle l\rangle}\right|^{2}|\leq| u_{l}-z_{l}^{\langle l\rangle} e^{-i \omega_{l} t}|| u_{l}+z_{l}^{\langle l\rangle} e^{-i \omega_{l} t} \mid \leq\left(\left|u_{l}-\tilde{u}_{l}\right|+\left|\tilde{u}_{l}-z_{l}^{\langle l\rangle} e^{-i \omega_{l} t}\right|\right)\left(\left|u_{l}\right|+\left|z_{l}^{\langle l\rangle}\right|\right) .
$$

With Proposition 3 this can be bounded by $C \varepsilon^{\frac{7}{2}}$. This yields the inequality stated in the proposition.

\subsection{From short to long time intervals}

By now we have proven Theorem 1 on the short time interval $\left[0, \varepsilon^{-1}\right]$. The extension to long time intervals $\left[0, \varepsilon^{-N}\right]$ as stated in the theorem can be done as in [6, Subsection 4.5]:

On intervals $\left[m \varepsilon^{-1},(m+1) \varepsilon^{-1}\right]$ for integers $m$ we consider the approximate solution of the modulation system given by Proposition 3 with initial data $u\left(\cdot, m \varepsilon^{-1}\right)$. On each of these intervals we have conservation of $\mathcal{I}_{\langle l\rangle}$ up to $\varepsilon^{N+3}$ by Lemma 4 (by "conservation" we mean conservation in the sense of this Lemma and Theorem 1). The difference of $\mathcal{I}_{\langle l\rangle}$ at the interfaces of these intervals is estimated by $\varepsilon^{N+3}$ using the second estimate of Lemma 3 and Propositions 3 and 4 . Hence $\mathcal{I}_{\langle l\rangle}$ is conserved up to $\varepsilon^{4}$ on an interval of length $\varepsilon^{-N}$ which consists of $\varepsilon^{-N+1}$ intervals of length $\varepsilon^{-1}$. By Lemma 5 this implies the conservation of the actions $I_{l}$ on this interval up to $\varepsilon^{\frac{7}{2}}$. This lemma also guarantees that the initial data $u\left(\cdot, m \varepsilon^{-1}\right)$ remains of size $\varepsilon$ on the considered short intervals for $\varepsilon$ small enough since $\|u\|_{s}^{2}=\sum_{l}\left|\omega_{l}\right|^{s} 2 I_{l}(u, \bar{u})$.

This concludes the proof of Theorem 1.

\section{$5 \quad$ Near-conservation of actions, energy and momentum for the semi-discretized nonlinear Schrödinger equation}

We now consider a spectral semi-discretization in space of the nonlinear Schrödinger equation (1) and study the actions (2) along solutions of the semi-discretized equation. Similar results could be obtained for a semi-discretization in space with finite differences.

\subsection{Spectral semi-discretization in space}

The semi-discretization in space is done by a spectral collocation method. As an ansatz for the solution $u$ of the nonlinear Schrödinger equation (1) we choose the trigonometric polynomial

$$
u^{M}(x, t)=\sum_{j \in \mathcal{M}} q_{j}(t) e^{i(j \cdot x)}
$$

where $\mathcal{M}=\{-M, \ldots, M-1\}^{d}$. We require this ansatz to fulfill (1) in the collocation points

$$
x_{k}=\frac{\pi}{M} k, \quad k \in \mathcal{M} .
$$

With $u^{M}\left(x_{k}, t\right)_{k \in \mathcal{M}}=\mathcal{F}_{2 M}\left(q_{j}(t)\right)_{j \in \mathcal{M}}$, where $\mathcal{F}_{2 M}$ denotes the $d$-dimensional discrete Fourier transform, we arrive at the system of ordinary differential equations

$$
i \frac{d u^{M}}{d t}\left(x_{k}, t\right)_{k \in \mathcal{M}}=\mathcal{F}_{2 M} \Omega \mathcal{F}_{2 M}^{-1} u^{M}\left(x_{k}, t\right)_{k \in \mathcal{M}}+\left(\left|u^{M}\left(x_{k}, t\right)\right|^{2} u^{M}\left(x_{k}, t\right)\right)_{k \in \mathcal{M}}
$$


where $\Omega=\operatorname{diag}\left(\left(\omega_{l}\right)_{l \in \mathcal{M}}\right)$ is the diagonal matrix with the frequencies $\omega_{l}, l \in \mathcal{M}$, on its diagonal. The initial value is $u^{M}\left(x_{k}, 0\right)_{k \in \mathcal{M}}=u\left(x_{k}, 0\right)_{k \in \mathcal{M}}$. We note that the semi-discretized system (24) is a finite dimensional complex Hamiltonian system with Hamiltonian $H_{M}\left(u^{M}\left(x_{k}, t\right)_{k \in \mathcal{M}},{\overline{u^{M}\left(x_{k}, t\right)}}_{k \in \mathcal{M}}\right)=$ $H_{M}\left(u^{M}, \overline{u^{M}}\right)$,

$$
\begin{aligned}
H_{M}\left(u^{M}, \overline{u^{M}}\right) & =\frac{1}{2}{\overline{u^{M}\left(x_{k}, t\right)}}_{k \in \mathcal{M}}^{T} \mathcal{F}_{2 M} \Omega \mathcal{F}_{2 M}^{-1} u^{M}\left(x_{k}, t\right)_{k \in \mathcal{M}}+\frac{1}{4} \sum_{k \in \mathcal{M}}\left|u^{M}\left(x_{k}\right)\right|^{4} \\
& =\frac{1}{2(2 \pi)^{d}} \int_{[-\pi, \pi]^{d}}\left(\left|\nabla u^{M}\right|^{2}+\left(V * u^{M}\right) \overline{u^{M}}+\frac{1}{2} \mathcal{Q}\left(\left|u^{M}\right|^{4}\right)\right) d x .
\end{aligned}
$$

Here, we use the notation $\mathcal{Q}(v)$ for the trigonometric interpolation of a periodic function $v=$ $\sum_{j \in \mathbb{Z}^{d}} v_{j} e^{i(j \cdot x)}$ in the collocation points, i. e.

$$
\mathcal{Q}(v)=\sum_{j \in \mathcal{M}}\left(\sum_{m \in \mathbb{Z}^{d}} v_{j+2 M m}\right) e^{i(j \cdot x)} .
$$

The semi-discretized equation (24) can be rewritten as

$$
i \frac{d u^{M}}{d t}=-\Delta u^{M}+V * u^{M}+\mathcal{Q}\left(\left|u^{M}\right|^{2} u^{M}\right)
$$

with initial value

$$
u^{M}(\cdot, 0)=\mathcal{Q}(u(\cdot, 0))
$$

\subsection{Statement of the result}

The nonlinear Schrödinger equation (1) is an infinite dimensional complex Hamiltonian system with Hamiltonian or total energy

$$
H(u, \bar{u})=\frac{1}{2(2 \pi)^{d}} \int_{[-\pi, \pi]^{d}}\left(|\nabla u|^{2}+(V * u) \bar{u}+\frac{1}{2}|u|^{4}\right) d x .
$$

Along solutions of (1) this Hamiltonian as well as the momentum

$$
K(u, \bar{u})=i \frac{1}{(2 \pi)^{d}} \int_{[-\pi, \pi]^{d}}(u \nabla \bar{u}-\bar{u} \nabla u) d x
$$

are exactly conserved. However, they are not exact invariants of the semi-discretized system (24). We now formulate our main result for this semi-discretized system which states that energy, momentum, and all actions (2) are approximately conserved along solutions of the semi-discretized system over long times.

We use notations similar to those of Section 2, equation (4), but now $\mathbf{k}=\left(k_{l}\right)_{l \in \mathcal{M}}$ and $\boldsymbol{\omega}=$ $\left(\omega_{l}\right)_{l \in \mathcal{M}}$ are finite sequences and

$$
j(\mathbf{k})=\sum_{l \in \mathcal{M}} k_{l} l \bmod 2 M \in \mathcal{M} .
$$

Here, mod $2 M$ denotes the reduction modulo $2 M$ of each component where the representative is chosen in $\mathcal{M}$. The set of near-resonant indices now consists of pairs $(j(\mathbf{k}), \mathbf{k})$ with a finite sequence $\mathbf{k}=\left(k_{l}\right)_{l \in \mathcal{M}}$ of integers,

$$
\mathcal{R}_{\varepsilon, M}=\left\{(j, \mathbf{k}): j=j(\mathbf{k}), \mathbf{k} \neq\langle j\rangle,\left|\mathbf{k} \cdot \boldsymbol{\omega}-\omega_{j}\right|<\varepsilon^{\frac{1}{2}},\|\mathbf{k}\| \leq 2 N+2\right\},
$$

and the non-resonance condition reads

$$
\sup _{(j, \mathbf{k}) \in \mathcal{R}_{\varepsilon, M}} \frac{\left|\omega_{j}\right|^{s-\frac{d+1}{2}}}{\left|\boldsymbol{\omega}^{\left(s-\frac{d+1}{2}\right)|\mathbf{k}|}\right|} \varepsilon^{\|\mathbf{k}\|+1} \leq C_{0} \varepsilon^{2 N+4}
$$

for a constant $C_{0}$ (independent of $\varepsilon$ ) and a given natural number $N$. The following theorem corresponds to Theorem 1. 
Theorem 2. For given $N$ and $s \geq d+1$ there exists $\varepsilon_{0}>0$ such that the following holds: Under the conditions of small initial data $\left\|u^{M}(\cdot, 0)\right\|_{s} \leq \varepsilon \leq \varepsilon_{0}$ and of non-resonance (29), the estimates

$$
\begin{aligned}
& \sum_{l \in \mathcal{M}}\left|\omega_{l}\right|^{s} \frac{\left|I_{l}\left(u^{M}(\cdot, t), \overline{u^{M}}(\cdot, t)\right)-I_{l}\left(u^{M}(\cdot, 0), \overline{u^{M}}(\cdot, 0)\right)\right|}{\varepsilon^{2}} \leq C \varepsilon^{\frac{3}{2}} \quad \text { for } 0 \leq t \leq \varepsilon^{-N}, \\
& \quad \frac{\left|H\left(u^{M}(\cdot, t), \overline{u^{M}}(\cdot, t)\right)-H\left(u^{M}(\cdot, 0), \overline{u^{M}}(\cdot, 0)\right)\right|}{\varepsilon^{2}} \leq C \varepsilon^{2} M^{-s} \quad \text { for } 0 \leq t \leq \varepsilon^{-N}, \\
& \sum_{r=1}^{d} \frac{\left|K_{r}\left(u^{M}(\cdot, t), \overline{u^{M}}(\cdot, t)\right)-K_{r}\left(u^{M}(\cdot, 0), \overline{u^{M}}(\cdot, 0)\right)\right|}{\varepsilon^{2}} \leq C \min \left(\varepsilon^{\frac{3}{2}}, t \varepsilon^{2} M^{-\left(s-\frac{d+1}{2}\right)}\right) \quad \text { for } 0 \leq t \leq \varepsilon^{-N}
\end{aligned}
$$

hold for solutions $u^{M}(x, t)$ of (26) with a constant $C$ which depends on $C_{0}, d, N, s$, and $V$ but is independent of $\varepsilon, M$, and $t$.

The proof of this theorem is given in the following section.

\section{Modulated Fourier expansions for the semi-discretized equation}

The proof of the near-conservation of actions in Theorem 2 is a modification of the proof of Theorem 1 given in Sections 3 and 4. We mainly state the differences.

\subsection{The modulation system and its approximate solution}

The ansatz for $u^{M}$ is chosen again as a modulated Fourier expansion

$$
\tilde{u}(x, t)=\sum_{\|\mathbf{k}\| \leq K} z_{j(\mathbf{k})}^{\mathbf{k}}(\varepsilon t) e^{i(j(\mathbf{k}) \cdot x)} e^{-i(\mathbf{k} \cdot \boldsymbol{\omega}) t}=\sum_{\|\mathbf{k}\| \leq K} z^{\mathbf{k}}(x, \varepsilon t) e^{-i(\mathbf{k} \cdot \boldsymbol{\omega}) t}
$$

with $z^{\mathbf{k}}(x, \varepsilon t)=z_{j(\mathbf{k})}^{\mathbf{k}}(\varepsilon t) e^{i(j(\mathbf{k}) \cdot x)}$ and $K=2 N+2$. Now and in the following $\mathbf{k}=\left(k_{l}\right)_{l \in \mathcal{M}}$ is a finite sequence of integers $k_{l}$ with $\|\mathbf{k}\| \leq K$. For the derivation of the modulation system we proceed similarly as in Section 3. We insert (30) in (26) and note that for $\mathbf{k}=\mathbf{k}^{1}+\mathbf{k}^{2}-\mathbf{k}^{3}$ we have

$$
\mathcal{F}_{j}\left(\mathcal{Q}\left(z^{\mathbf{k}^{1}} z^{\mathbf{k}^{2}} \overline{z^{\mathbf{k}^{3}}}\right)\right)= \begin{cases}z_{j\left(\mathbf{k}^{1}\right)}^{\mathbf{k}^{1}} z_{j\left(\mathbf{k}^{2}\right)}^{\mathbf{k}^{2}} \overline{z^{\mathbf{k}^{3}\left(\mathbf{k}^{3}\right)}}, & j=j(\mathbf{k}) \\ 0, & \text { else }\end{cases}
$$

since $j(\mathbf{k}) \equiv j\left(\mathbf{k}^{1}\right)+j\left(\mathbf{k}^{2}\right)-j\left(\mathbf{k}^{3}\right)(\bmod 2 M)$ and $j(\mathbf{k}) \in \mathcal{M}$. Comparing the coefficients of $e^{i(j(\mathbf{k}) \cdot x)} e^{-i(\mathbf{k} \cdot \boldsymbol{\omega}) t}$ gives

$$
i \varepsilon \dot{z}_{j(\mathbf{k})}^{\mathbf{k}}+(\mathbf{k} \cdot \boldsymbol{\omega}) z_{j(\mathbf{k})}^{\mathbf{k}}=\omega_{j(\mathbf{k})} z_{j(\mathbf{k})}^{\mathbf{k}}+\sum_{\mathbf{k}^{1}+\mathbf{k}^{2}-\mathbf{k}^{3}=\mathbf{k}} \mathcal{F}_{j(\mathbf{k})}\left(\mathcal{Q}\left(z^{\mathbf{k}^{1}} z^{\mathbf{k}^{2}} \overline{z^{\mathbf{k}^{3}}}\right)\right)
$$

The only difference in comparison with the continuous modulation system (7a) is the presence of $\mathcal{Q}$. For the initial condition we obtain

$$
\sum_{\mathbf{k}} z_{j}^{\mathbf{k}}(0)=u_{j}^{M}(0)
$$

For this modulation system we have an approximate solution $\mathbf{z}$ as in Proposition 3 with the same estimates and constants independent of $\varepsilon, M$, and $0 \leq t \leq \varepsilon^{-1}$ (and $u$ replaced by $u^{M}$ and $j \in \mathbb{Z}^{d}$ replaced by $j \in \mathcal{M}$ ). To see this, we only have to ensure that the presence of $\mathcal{Q}$ in $(31 \mathrm{a})$ does not affect the estimates of the nonlinearity. This is done by the first inequality of the following lemma which is similar to [10, Lemma 4.2] (but here in arbitrary spatial dimension). 
Lemma 6. For $s>\frac{d}{2}$ and $s^{\prime} \geq 0$ we have

$$
\left\|\sum_{\mathbf{k}}\left\{\mathcal{Q}\left(c^{\mathbf{k}}\right)\right\}\right\|_{s} \leq C\left\|\sum_{\mathbf{k}}\left\{c^{\mathbf{k}}\right\}\right\|_{s} \quad \text { and } \quad\|\mathcal{Q}(v)-v\|_{s^{\prime}} \leq C M^{-\left(s-s^{\prime}\right)}\|v\|_{s}
$$

with a constant $C$ which depends on $d, s$, and $V$ but not on $\varepsilon$ and $M$.

Proof. Recall from Lemma 1 that $\sum_{k \in \mathbb{Z}^{d}} \frac{1}{\left|\omega_{k}\right|^{s}}$ converges for $s>\frac{d}{2}$. This implies that

$$
M^{2 s} \sum_{0 \neq m \in \mathbb{Z}^{d}} \frac{1}{\left|\omega_{j+2 M m}\right|^{s}} \leq C
$$

for a constant $C$ which depends on $d, s$, and $V$ but not on $\varepsilon, j$, and $M$.

We have

$$
\left\|\sum_{\mathbf{k}}\left\{\mathcal{Q}\left(c^{\mathbf{k}}\right)\right\}\right\|_{s}^{2} \leq \sum_{j \in \mathcal{M}}\left|\omega_{j}\right|^{s}\left(\sum_{m \in \mathbb{Z}^{d}} \sum_{\mathbf{k}}\left|c_{j+2 M m}^{\mathbf{k}}\right|\right)^{2}=\left\|\sum_{\mathbf{k}} \mathcal{Q}\left(\left\{c^{\mathbf{k}}\right\}\right)\right\|_{s}^{2}
$$

As in the proof of [10, Lemma 4.2] we have with the Cauchy-Schwarz inequality

$$
\|\mathcal{Q} v\|_{s}^{2} \leq \sum_{j \in \mathcal{M}}\left(\sum_{m \in \mathbb{Z}^{d}} \frac{\left|\omega_{j}\right|^{s}}{\left|\omega_{j+2 M m}\right|^{s}}\right) \sum_{m \in \mathbb{Z}^{d}}\left|\omega_{j+2 M m}\right|^{s}\left|v_{j+2 M m}\right|^{2} .
$$

The term $\sum_{m \in \mathbb{Z}^{d}} \frac{\left|\omega_{j}\right|^{s}}{\left|\omega_{j+2 M m}\right|^{s}}$ can be estimated independently of $j$ with (32) concluding the proof of the first estimate of the lemma.

As in the proof of [10, Lemma 4.2] we further have

$$
\begin{aligned}
\|\mathcal{Q}(v)-v\|_{s^{\prime}}^{2} \leq & \sum_{j \notin \mathcal{M}}\left|\omega_{j}\right|^{-\left(s-s^{\prime}\right)}\left|\omega_{j}\right|^{s}\left|v_{j}\right|^{2} \\
& +\sum_{j \in \mathcal{M}}\left(\sum_{0 \neq m \in \mathbb{Z}^{d}} \frac{\left|\omega_{j}\right|^{s^{\prime}}}{\left|\omega_{j+2 M m}\right|^{s}}\right)\left(\sum_{0 \neq m \in \mathbb{Z}^{d}}\left|\omega_{j+2 M m}\right|^{s}\left|v_{j+2 M m}\right|^{2}\right) .
\end{aligned}
$$

With (32) this can be estimated by $C M^{-2\left(s-s^{\prime}\right)}\|v\|^{2}$.

\subsection{Near-conservation of actions}

Let

$$
U(\mathbf{z})=\sum_{\mathbf{k}^{1}+\mathbf{k}^{2}-\mathbf{k}^{3}-\mathbf{k}^{4}=\mathbf{0}} \frac{1}{(2 \pi)^{d}} \int_{\mathbb{T}^{d}} \mathcal{Q}\left(z^{\mathbf{k}^{1}} z^{\mathbf{k}^{2}} \overline{z^{\mathbf{k}^{3}} z^{\mathbf{k}^{4}}}\right) d x .
$$

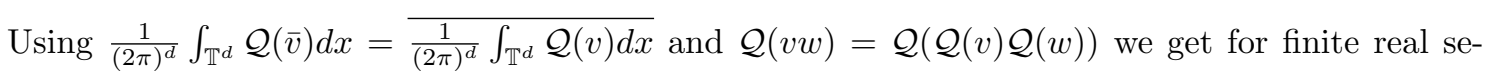
quences $\boldsymbol{\mu}=\left(\mu_{l}\right)_{l \in \mathcal{M}}$

$$
\begin{aligned}
0= & \left.\frac{d}{d \theta}\right|_{\theta=0} U\left(\left(e^{i(\mathbf{k} \cdot \boldsymbol{\mu}) \theta} z^{\mathbf{k}}\right)_{\mathbf{k}}\right)=\sum_{\mathbf{k}} \frac{1}{(2 \pi)^{d}} \int_{\mathbb{T}^{d}} 2 i(\mathbf{k} \cdot \boldsymbol{\mu}) \mathcal{Q}\left(z^{\mathbf{k}} \sum_{\mathbf{k}^{2}-\mathbf{k}^{3}-\mathbf{k}^{4}=-\mathbf{k}} z^{\mathbf{k}^{2}} \overline{z^{\mathbf{k}^{3}} z^{\mathbf{k}^{4}}}\right) d x \\
& +\sum_{\mathbf{k}} \frac{1}{(2 \pi)^{d}} \int_{\mathbb{T}^{d}} 2(-i)(\mathbf{k} \cdot \boldsymbol{\mu}) \mathcal{Q}\left(\overline{z^{\mathbf{k}}} \sum_{\mathbf{k}^{1}+\mathbf{k}^{2}-\mathbf{k}^{3}=\mathbf{k}} z^{\mathbf{k}^{1}} z^{\mathbf{k}^{2}} \overline{z^{\mathbf{k}^{3}}}\right) d x \\
=- & \operatorname{Re}\left(\sum_{\mathbf{k}} i(\mathbf{k} \cdot \boldsymbol{\mu}) \sum_{j \in \mathcal{M}} \overline{z_{j}^{\mathbf{k}}} \mathcal{F}_{j}\left(\mathcal{Q}\left(\sum_{\mathbf{k}^{1}+\mathbf{k}^{2}-\mathbf{k}^{3}=\mathbf{k}} z^{\mathbf{k}^{1}} z^{\mathbf{k}^{2}} \overline{z^{\mathbf{k}^{3}}}\right)\right)\right),
\end{aligned}
$$

and with (31a) and $2 \operatorname{Re}\left(\overline{z_{j}^{\mathbf{k}}} \varepsilon \dot{z}_{j}^{\mathbf{k}}\right)=\frac{d}{d t}\left|z_{j}^{\mathbf{k}}\right|^{2}$ we obtain

$$
0=2 \frac{d}{d t} \sum_{\mathbf{k}}(\mathbf{k} \cdot \boldsymbol{\mu})\left|z_{j(\mathbf{k})}^{\mathbf{k}}\right|^{2}+4 \operatorname{Re}\left(\sum_{\mathbf{k}} i(\mathbf{k} \cdot \boldsymbol{\mu}) \overline{z_{j(\mathbf{k})}^{\mathbf{k}}} d_{j(\mathbf{k})}^{\mathbf{k}}\right)
$$


Hence

$$
\mathcal{I}_{\boldsymbol{\mu}}(\mathbf{z}(\cdot, \varepsilon t))=\frac{1}{2} \sum_{\mathbf{k}}(\mathbf{k} \cdot \boldsymbol{\mu})\left|z_{j(\mathbf{k})}^{\mathbf{k}}(\varepsilon t)\right|^{2}
$$

is an almost-invariant of the modulation system (31a).

For the estimation of $\sum_{l \in \mathcal{M}}\left|\omega_{l}\right|^{s}\left|\frac{d}{d t} \mathcal{I}_{\langle l\rangle}(\cdot, \varepsilon t)\right|$ we need Lemma 3 for the semi-discrete setting. In the proof of this lemma we concluded $|j(\mathbf{k})|>\frac{1}{2}|L|$ from $\left|\sum_{l \in \mathbb{Z}^{d}} k_{l} l\right|>\frac{1}{2}|L|$. However, this conclusion is not true any more $\left(\right.$ recall $\left.j(\mathbf{k})=\sum_{l \in \mathbb{Z}^{d}} k_{l} l \bmod 2 M\right)$. We therefore adapt this part of the proof of Lemma 3.

Adapted proof of Lemma 3. We need to (re)bound

$$
\sup _{(j, \mathbf{k}) \in \mathcal{S}_{\varepsilon, M}} \frac{\sum_{l \in \mathcal{M}}\left|k_{l}\right|\left|\omega_{l}\right|^{s}}{\left|\boldsymbol{\omega}^{\left(s-\frac{d+1}{2}\right)|\mathbf{k}|}\right|\left|\omega_{j}\right|^{\frac{d+1}{2}}}
$$

where $\mathcal{S}_{\varepsilon, M}=\left\{(j, \mathbf{k}): j \neq j(\mathbf{k}), \mathbf{k} \neq\langle j\rangle,(j, \mathbf{k}) \notin \mathcal{R}_{\varepsilon, M},\|\mathbf{k}\| \leq K\right\}$. For this purpose let again $(j, \mathbf{k})=(j(\mathbf{k}), \mathbf{k}) \in \mathcal{S}_{\varepsilon, M}$. Let $|\cdot|_{\max }$ be the maximum norm on $\mathbb{R}^{d}$ which is equivalent to the Euclidean norm $|\cdot|$,

$$
|l|^{2} \leq d|l|_{\max }^{2} \leq d|l|^{2} .
$$

Using this and the asymptotics of the frequencies we have $\sum_{l \in \mathcal{M}}\left|k_{l}\right|\left|\omega_{l}\right|^{s} \leq C\left|\omega_{L}\right|^{s}$ if $L \in \mathcal{M}$ is the index of largest norm $|\cdot|_{\max }$ with $k_{L} \neq 0$. The constant $C$ depends only on $d, K, s$, and $V$. For $\left|k_{L}\right|>1$ or $k_{l} \neq 0$ for $l \neq L$ with $|l|_{\max }>\frac{1}{2 K}|L|_{\max }$ we have using $s \geq d+1$ and (33) $\left|\boldsymbol{\omega}^{\left(s-\frac{d+1}{2}\right)|\mathbf{k}|}\right| \geq C\left|\omega_{L}\right|^{s-\frac{d+1}{2}}\left|\omega_{L}\right|^{\frac{d+1}{2}}$ for a constant which depends on $d, K, s$, and $V$. For the other $\mathbf{k}$ we have

$$
\frac{3}{2}|L|_{\max }>|L|_{\max }+\frac{K-1}{2 K}|L|_{\max } \geq\left|k_{L} L+\sum_{|l| \leq \frac{1}{2 K}|L|} k_{l} l\right|_{\max }=\left|\sum_{l \in \mathbb{Z}^{d}} k_{l} l\right|_{\max }>\frac{1}{2}|L|_{\max } .
$$

From this we can conclude $|j(\mathbf{k})|_{\max } \geq \frac{1}{2}|L|_{\max }$. Hence, we get with (33) $\left|\boldsymbol{\omega}^{\left(s-\frac{d+1}{2}\right)|\mathbf{k}|}\right|\left|\omega_{j}\right|^{\frac{d+1}{2}} \geq$ $C\left|\omega_{L}\right|^{s-\frac{d+1}{2}}\left|\omega_{L}\right|^{\frac{d+1}{2}}$ with a constant which depends on $d, s$, and $V$.

So, Lemma 3 is true in the semi-discretized situation with constants independent of $M$. Now, the approximate conservation of actions along solutions of the semi-discretized equation can be shown as in Section 4.

\subsection{Near-conservation of energy}

The conservation of actions as stated in Theorem 2 implies $\left|\left\|u^{M}(\cdot, t)\right\|^{2}-\left\|u^{M}(\cdot, 0)\right\|^{2}\right| \leq C \varepsilon^{\frac{7}{2}}$ and hence

$$
\left\|u^{M}(\cdot, t)\right\|_{s} \leq 2 \varepsilon
$$

for times $0 \leq t \leq \varepsilon^{-N}$ provided that $\varepsilon$ is sufficiently small. This spatial regularity and the Hamiltonian structure of the semi-discrete system are the main tools to prove the long-time nearconservation of energy and momentum with the arguments of [10, Section 6].

We have from (25) and (27)

$$
H\left(u^{M}(\cdot, t), \overline{u^{M}}(\cdot, t)\right)-H_{M}\left(u^{M}(\cdot, t), \overline{u^{M}}(\cdot, t)\right)=\frac{1}{4(2 \pi)^{d}} \int_{[-\pi, \pi]^{d}}\left(\left|u^{M}(\cdot, t)\right|^{4}-\mathcal{Q}\left(\left|u^{M}(\cdot, t)\right|^{4}\right)\right) d x
$$

As in $[10$, Subsection 6.2$]$ the right-hand side can be bounded using Lemma 6 with $s^{\prime}=0$ by

$$
C M^{-s}\left\|\left|u^{M}(\cdot, t)\right|^{4}\right\|_{s} \leq C M^{-s} \varepsilon^{4}
$$

for $0 \leq t \leq \varepsilon^{-N}$ by (34). This implies the long-time near-conservation of energy as stated in Theorem 2 since the Hamiltonian $H_{M}$ is exactly conserved along solutions $u^{M}$ of (24). 


\subsection{Near-conservation of momentum}

Let $v(x, t)$ be the exact solution of (1) with initial value $v(\cdot, 0)=u^{M}(\cdot, 0)$. Along this solution the momentum (28), whose $r$-th component is

$$
K_{r}(u, \bar{u})=i \frac{1}{(2 \pi)^{d}} \int_{[-\pi, \pi]^{d}}\left(u \frac{d \bar{u}}{d x_{r}}-\bar{u} \frac{d u}{d x_{r}}\right) d x=2 \sum_{j \in \mathbb{Z}^{d}} j_{r}\left|u_{j}\right|^{2},
$$

is exactly conserved. With the arguments from Subsection 3.8 we get $\|v(\cdot, t)\|_{s} \leq C \varepsilon$ for $0 \leq t \leq$ $\varepsilon^{-1}$. For the difference $v-u^{M}$ we have

$$
i\left(v_{j}-u_{j}^{M}\right)_{t}=\omega_{j}\left(v_{j}-u_{j}^{M}\right)+\mathcal{F}_{j}\left(\mathcal{Q}\left(|v|^{2} v-\left|u^{M}\right|^{2} u^{M}\right)\right)+\mathcal{F}_{j}\left(|v|^{2} v-\mathcal{Q}\left(|v|^{2} v\right)\right) .
$$

With the second inequality of Lemma 6 with $s^{\prime}=\frac{d+1}{2}$ and Lemma 1 the last term is bounded by $\left\||v|^{2} v-\mathcal{Q}\left(|v|^{2} v\right)\right\|_{\frac{d+1}{2}} \leq C M^{-\left(s-\frac{d+1}{2}\right)} \varepsilon^{3}$. The arguments from Subsection 3.8 applied to (35) yield

$$
\left\|v(\cdot, t)-u^{M}(\cdot, t)\right\|_{\frac{d+1}{2}} \leq C t \varepsilon^{3} M^{-\left(s-\frac{d+1}{2}\right)}
$$

for $0 \leq t \leq \varepsilon^{-1}$. This implies using in addition Proposition 3

$$
\left|K_{r}\left(u^{M}(\cdot, t), \overline{u^{M}}(\cdot, t)\right)-K_{r}(v(\cdot, t), \bar{v}(\cdot, t))\right| \leq C t \varepsilon^{4} M^{-\left(s-\frac{d+1}{2}\right)}
$$

for $0 \leq t \leq \varepsilon^{-1}$. The extension to long time intervals can be done as in [10, Subsection 6.1] which yields

$$
\left|K_{r}\left(u^{M}(\cdot, t), \overline{u^{M}}(\cdot, t)\right)-K_{r}\left(u^{M}(\cdot, 0), \overline{u^{M}}(\cdot, 0)\right)\right| \leq C t \varepsilon^{4} M^{-\left(s-\frac{d+1}{2}\right)}
$$

for $0 \leq t \leq \varepsilon^{-N}$. On the other hand we have using the conservation of actions

$$
\begin{aligned}
\mid K_{r}\left(u^{M}(\cdot, t), \overline{u^{M}}(\cdot, t)\right) & -K_{r}\left(u^{M}(\cdot, 0), \overline{u^{M}}(\cdot, 0)\right) \mid \\
& \leq 2 \sum_{j \in \mathcal{M}}\left|j_{r}\right|\left|I_{j}\left(u^{M}(\cdot, t), \overline{u^{M}}(\cdot, t)\right)-I_{j}\left(u^{M}(\cdot, 0), \overline{u^{M}}(\cdot, 0)\right)\right| \leq C \varepsilon^{\frac{7}{2}}
\end{aligned}
$$

for $0 \leq t \leq \varepsilon^{-N}$ and $r=1, \ldots, d$ since $\left|j_{r}\right| \leq C\left|\omega_{j}\right|^{s}$. This concludes the proof of Theorem 2 .

\section{Conclusion and comparison}

In this paper the long-time near-conservation of actions has been shown for the nonlinear Schrödinger equation (1) and its spectral semi-discretization (24) in the weakly nonlinear setting of small initial data, over time scales far beyond a linear perturbation analysis. The implied spatial regularity allowed to show the long-time near-conservation of energy and momentum for the semidiscretized system. These results have to be compared with [6, Theorem 1] and [10, Theorems $3.1,3.2,3.3]$ where the corresponding quantities of semilinear wave equations and their spectral semi-discretizations are studied.

The method of proof presented in the present paper is the same as in [6] and [10]: A modulated Fourier expansion of the solution of (1) or its semi-discretization (24) is established, and the coefficients of this expansion are determined up to a small defect on a time interval of length $\varepsilon^{-1}$ (Sections 3 and 6.1). The system determining these coefficients has invariants which are conserved up to $\varepsilon^{-N+3}$ on a time interval of length $\varepsilon^{-1}$ and which are close to the actions (Sections 4 and 6.2 ). The result for the long time interval of length $\varepsilon^{-N}$ is obtained by patching together the short time intervals.

However, there are some remarkable differences between the semilinear wave equation and the nonlinear Schrödinger equation, which we collect in the following.

A major difference lies in the validity for arbitrary spatial dimension of Theorems 1 and 2 for the nonlinear Schrödinger equation (so far, the results for semilinear wave equations have only been established for the one dimensional problem). This is due to the fact that the frequencies of the Schrödinger equation behave milder than those of the wave equation in arbitrary dimension, 
cf. [3] where a theorem similar to Theorem 1 is shown by transforming (1) to a normal form. To make use of this fact in the context of modulated Fourier expansions, it is essential to observe that it suffices to consider modulation functions consisting of a single wave, cf. Proposition 2.

Another difference in the statements of the results is that the actions divided by $\varepsilon^{2}$ are conserved up to $\varepsilon^{\frac{3}{2}}$ for the nonlinear Schrödinger equation (instead of $\varepsilon$ for the semilinear wave equation). This is explained by the cubic (instead of quadratic) nonlinearity in (1). For this reason, one could even expect a conservation up to $\varepsilon^{2}$, but it is not clear how to achieve $\varepsilon^{2}$. Note however that in the near-conservation of energy and momentum divided by $\varepsilon^{2}$ along solutions of the semidiscretized equation we get a factor $\varepsilon^{2}$ as expected since these proofs only rely on the spatial regularity established by the conservation of actions. In [6], the enhancement from $\varepsilon^{\frac{1}{2}}$ to $\varepsilon$ in the conservation of actions is derived by bounding the denominator $\left|\omega_{j}^{2}-(\mathbf{k} \cdot \boldsymbol{\omega})^{2}\right|$ from below independently of $\varepsilon$ for (amongst others) $\mathbf{k}= \pm\langle k\rangle \pm\langle l\rangle$ and $j=k+l$, cf. equation (30) and the proof of Theorem 4 in [6]. Here, we should accomplish this for $\mathbf{k}= \pm\langle k\rangle \pm\langle l\rangle \pm\langle m\rangle$ due to the cubic nonlinearity, but this is not true. However, the conservation of the actions in Theorems 1 and 2 can be enhanced to $\varepsilon^{2-\frac{1}{m}}$ for any integer $m \geq 2$ with a new definition of the set of near-resonant indices

$$
\tilde{\mathcal{R}}_{\varepsilon}=\left\{(j, \mathbf{k}): j=j(\mathbf{k}), \mathbf{k} \neq\langle j\rangle,\left|\mathbf{k} \cdot \boldsymbol{\omega}-\omega_{j}\right|<\varepsilon^{\frac{1}{m}},\|\mathbf{k}\| \leq 2 N+2\right\} .
$$

Then we get $\beta_{n} \leq C \varepsilon^{1-\frac{1}{m}}$ instead of $\beta_{n} \leq C \varepsilon^{\frac{1}{2}}$ in Subsection 3.6, and this yields $\varepsilon^{4-\frac{1}{m}}$ instead of $\varepsilon^{\frac{7}{2}}$ in Lemma 5 .

Due to the cubic nonlinearity a linear perturbation analysis allows estimates over times $\varepsilon^{2}$. This suggests to consider the time scale $\tau=\varepsilon^{2} t$ instead of $\tau=\varepsilon t$ for the coefficients $z_{j}^{\mathbf{k}}$ of the modulated Fourier expansion. However, this time scale leads to difficulties in the estimations of the defect in Subsection 3.7 since a result corresponding to [6, equation (22)] is not true for the cubic nonlinearity. The usage of the time scale $\varepsilon t$ in combination with the cubic nonlinearity even simplifies the estimation of the defect in the modulation functions in comparison with $[6$, Subsection 3.11]: Yet another rescaling of the variables as in the estimation of the defect in [6] is no longer necessary.

The technical differences arising in the analysis of the modulation functions are mainly caused by a different structure of the wave and the Schrödinger equation. While the wave equation is a second order partial differential equation with asymptotically linear frequencies, the Schrödinger equation is of first order with asymptotically quadratic frequencies. This leads to different linear parts in the modulation systems. For the nonlinear Schrödinger equation, this linear part is $\left(\mathbf{k} \cdot \boldsymbol{\omega}-\omega_{j(\mathbf{k})}\right) z_{j(\mathbf{k})}^{\mathbf{k}}($ equation $(7 \mathrm{a}))$ whereas it reads

$$
\left((\mathbf{k} \cdot \boldsymbol{\omega})^{2}-\omega_{j}^{2}\right) z_{j}^{\mathbf{k}}=\left(|\mathbf{k} \cdot \boldsymbol{\omega}|+\omega_{j}\right)\left(|\mathbf{k} \cdot \boldsymbol{\omega}|-\omega_{j}\right) z_{j}^{\mathbf{k}}
$$

for the semilinear wave equation (equation (15) in [6]). Hence, in the case of the wave equation $\left(|\mathbf{k} \cdot \boldsymbol{\omega}|+\omega_{j}\right) b_{j}^{\mathbf{k}}$ can be estimated and not only $b_{j}^{\mathbf{k}}$ as for the Schrödinger equation.

The first consequence is that in the proof of Lemma 3 we no longer have the factor $|\mathbf{k} \cdot \boldsymbol{\omega}|+1$ in the denominator of (23), but the absence of this term can be compensated by our knowledge that the modulation functions consist of single waves.

The second more fundamental consequence is that we need to use a different norm for the analysis of the modulation functions. Our mixture between $l^{2}$ - and $l^{1}$-framework described by (8) replaces the $l^{2}$-framework of $[6]$ where the norm

$$
\left(\sum_{j} \omega_{j}^{2 s} \sum_{\mathbf{k}}\left|z_{j}^{\mathbf{k}}\right|^{2}\right)^{\frac{1}{2}}
$$

is used instead. With this norm from [6] another rescaling of the variables is needed for the estimation of the nonlinearity, namely $c^{\mathbf{k}}=\boldsymbol{\omega}^{|\mathbf{k}|} \varepsilon^{-[[\mathbf{k}]]} z^{\mathbf{k}}$ with a slightly different definition of $[[\mathbf{k}]]$ due to the quadratic nonlinearity, cf. [6, Subsection 3.5] (recall that we scaled the variables by introducing $\left.c^{\mathbf{k}}=\varepsilon^{-[[\mathbf{k}]]} z^{\mathbf{k}}\right)$. This rescaling introduces a factor $\left(\omega_{j}-\mathbf{k} \cdot \boldsymbol{\omega}\right)$ before $b_{j}^{\mathbf{k}}$ in the modulation system for the initial values in [6] (to be more precise, it is responsible for the $\omega_{j}$ in this factor), cf. [6, Subsection 3.6]. This is no problem since $\left(|\mathbf{k} \cdot \boldsymbol{\omega}|+\omega_{j}\right) b_{j}^{\mathbf{k}}$ can be estimated. In the case of the nonlinear Schrödinger equation a factor $\omega_{j}$ would appear before $b_{j}^{\mathbf{k}}$ in the formula 
(10) for $a_{j}^{\langle j\rangle}(0)$ which we cannot handle by this argument. For this reason we choose the norm (8). A further advantage of this norm is that it simplifies many estimations in the analysis of the modulation functions.

\section{Acknowledgement}

This work was supported by DFG, Project LU 532/4-1.

\section{References}

[1] R. A. Adams. Sobolev spaces. Academic Press, New York-London, 1975. Pure and Applied Mathematics, Vol. 65.

[2] V. I. Arnold. Geometrical methods in the theory of ordinary differential equations, volume 250 of Grundlehren der Mathematischen Wissenschaften. Springer-Verlag, New York, 1983. Translated from the Russian by Joseph Szücs, Translation edited by Mark Levi.

[3] D. Bambusi and B. Grébert. Birkhoff normal form for partial differential equations with tame modulus. Duke Math. J., 135(3):507-567, 2006.

[4] J. Bourgain. Quasi-periodic solutions of Hamiltonian perturbations of 2D linear Schrödinger equations. Ann. of Math. (2), 148(2):363-439, 1998.

[5] D. Cohen, E. Hairer, and C. Lubich. Conservation of energy, momentum and actions in numerical discretizations of nonlinear wave equations. To appear in Numer. Math., 2008.

[6] D. Cohen, E. Hairer, and C. Lubich. Long-time analysis of nonlinearly perturbed wave equations via modulated Fourier expansions. Arch. Ration. Mech. Anal., 187(2):341-368, 2008.

[7] L. H. Eliasson and S. B. Kuksin. KAM for the non-linear Schrödinger equation. To appear in Ann. of Math., 2008.

[8] L. Gauckler and C. Lubich. Splitting integrators for nonlinear Schrödinger equations over long times. Preprint, http://na.uni-tuebingen.de/preprints.shtml, 2008.

[9] E. Hairer and C. Lubich. Long-time energy conservation of numerical methods for oscillatory differential equations. SIAM J. Numer. Anal., 38(2):414-441 (electronic), 2000.

[10] E. Hairer and C. Lubich. Spectral semi-discretisations of weakly nonlinear wave equations over long times. To appear in Found. Comput. Math., 2008.

[11] E. Hairer, C. Lubich, and G. Wanner. Geometric numerical integration. Structure-preserving algorithms for ordinary differential equations, volume 31 of Springer Series in Computational Mathematics. Springer-Verlag, Berlin, second edition, 2006. 